\title{
Short- and long-range interactions in the HIV-1 5'UTR regulate genome dimerization and packaging
}

Redmond Smyth ( $\square$ redmond.smyth@helmholtz-hiri.de )

Helmholtz Institute for RNA-based Infection Research https://orcid.org/0000-0002-1580-0671

\section{Liqing Ye}

Helmholtz Institute for RNA-based Infection Research

Anne-Sophie Gribling

Helmholtz Institute for RNA-based Infection Research

Patrick Bohn

Helmholtz Institute for RNA-based Infection Research

Anuja Kibe

Helmholtz Institute for RNA-based Infection Research

Charlene Börtlein

Helmholtz Institute for RNA-based Infection Research

Uddhav Ambi

Helmholtz Institute for RNA-based Infection Research

Shazeb Ahmad

Helmholtz Institute for RNA-based Infection Research

Marco Olguin-Nava

Helmholtz Institute for RNA-based Infection Research

Maureen Smith

Robert Koch-Institute

Neva Caliskan

Helmholtz Institute for RNA-based Infection Research

Max von Kleist

Robert Koch-Institute

Article

Keywords:

Posted Date: December 10th, 2021

DOI: https://doi.org/10.21203/rs.3.rs-613603/v1 
License: (c) (i) This work is licensed under a Creative Commons Attribution 4.0 International License. Read Full License

Version of Record: A version of this preprint was published at Nature Structural \&amp; Molecular Biology on March 28th, 2022. See the published version at https://doi.org/10.1038/s41594-022-00746-2. 


\section{Short and long-range interactions in the HIV-1 5'UTR regulate genome}

\section{2 dimerization and packaging}

3 Liqing $\mathrm{Ye}^{1}$, Anne-Sophie Gribling-Burrer ${ }^{1 \S}$, Patrick Bohn ${ }^{1 \S}$, Anuja Kibe ${ }^{1}$, Charlene Börtlein ${ }^{1}$, Uddhav

4 B Ambi ${ }^{1}$, Shazeb Ahmad ${ }^{1}$, Marco Olguin-Nava ${ }^{1}$, Maureen Smith ${ }^{3}$, Neva Caliskan ${ }^{1,2}$, Max von

5 Kleist $^{3}$, Redmond P. Smyth ${ }^{1,2}$

$6{ }^{1}$ Genome Architecture and Evolution of RNA viruses, Helmholtz Institute for RNA-based Infection

7 Research (HIRI), Würzburg, Germany

$8 \quad{ }^{2}$ Faculty of Medicine, University of Würzburg, Würzburg, Germany

$9 \quad{ }^{3}$ P5 Systems Medicine of Infectious Disease, Robert Koch-Institute, Berlin, Germany 


\section{Abstract}

13 Genome dimerization is a conserved feature of retroviral replication and a critical step in the HIV-1

14 life cycle, but how it is regulated is incompletely understood. Here, we developed FARS-seq

15 (Functional Analysis of RNA Structure) to comprehensively identify sequences and structures

16 within the HIV-1 5'UTR influencing dimerization. We found nucleotides important for dimerization

17 throughout the HIV-1 5'UTR and identified distinct structural conformations in monomeric and

18 dimeric RNA. The dimer displayed TAR, PolyA, PBS, and SL1-SL3 as stem-loops. In the monomer,

19 SL1 was dramatically reconfigured into long- and short-range base-pairings with polyA and PBS,

20 respectively. The polyA-SL1 interaction disrupts the major packaging motifs, and the PBS-SL1

21 interaction functionally couples the primer binding site with dimerization and $\operatorname{Pr} 55^{\text {Gag }}$ binding.

22 Altogether, our data provide insights into late stages of HIV-1 life cycle and a mechanistic

23 explanation for the link between RNA dimerization and packaging. 
HIV-1, like all retroviruses, packages two copies of its genome into viral particles. These genomes are non-covalently associated at an RNA motif called the dimerization initiation site (DIS). This association, known as dimerization, impacts multiple steps of the HIV-1 life cycle ${ }^{1,2}$. Dimerization is assumed to be a prerequisite for genome packaging into virions, although the mechanistic relationship between dimerization and packaging is still under debate ${ }^{3-6}$. It also plays a role in genome integrity and evolution by bringing two genomes in close proximity for strand-switch recombination ${ }^{3,7-10}$. Finally, it is linked to a structural switch that may regulate genome packaging and translation within cells ${ }^{5,11-16}$ (Fig. 1a). Despite its importance to HIV-1 biology, the RNA signals that regulate genome dimerization, and the molecular mechanism leading to the selection of the genome dimer from monomer for packaging are incompletely understood.

An extensive body of work maps the DIS to stem-loop 1 (SL1) of the HIV-1 5'UTR ${ }^{17-19}$ (Fig. 1b). SL1 contains a six-nucleotide long GC-rich palindromic sequence that initiates dimerization through an inter-molecular "kissing loop" interaction ${ }^{20-22}$. Although SL1 is widely considered the primary dimerization motif, numerous studies indicate that genome dimerization is also modulated by sequences outside of $\mathrm{SL} 1^{4,17,18,23-26}$. For example, dimerization is promoted by a long-range base pairing between nucleotides overlapping the gag start codon (AUG) and the unique $5^{\prime}$ element $(\mathrm{U} 5)^{12,26,27}$ (Fig. 1b). Alternatively, it is inhibited when the region containing the AUG folds into a small hairpin, in turn freeing U5 to form a pseudoknot interaction with $S L 1^{12,28}$ (Fig. 1b). The U5SL1 pseudoknot interaction was originally proposed as a liable interaction between the loop region of SL1 and U5, but a recent NMR study uncovered a more extensive base-pairing between U5 and $\mathrm{SL} 1^{13,14,28}$. Furthermore, intrinsic transcriptional start site heterogeneity, which produces transcript variants beginning with different counts of $G$ residues (1G, $2 G$ or $3 G$ ), has been shown to regulate dimerization by shifting the equilibrium between mutually exclusive structures containing either an U5-AUG, or a U5-SL1 interaction ${ }^{13,14,29:} 1 \mathrm{G}$ transcripts expose the DIS for dimerization and sequester the 5' cap, whereas $3 \mathrm{G}$ variants conceal the DIS whilst exposing the cap to enhance translation ${ }^{14}$. In addition to the U5-AUG and U5-SL1 conformations, over 20 structural models of 
the HIV-1 genome have been proposed, suggesting that the 5'UTR may dynamically adopt multiple conformational states ${ }^{30,31}$. It seems therefore likely that other structural forms of the HIV-1 genome exist to regulate genome dimerization, or other critical aspects of HIV-1 biology.

Sequences required for dimerization largely overlap with other conserved functional elements, such as those involved with genome packaging. Indeed, this genetic overlap between dimerization and packaging signals is a major reason why dimerization is considered to be a pre-requisite for packaging, even though the precise molecular mechanism underlying this phenomenon is unclear. In this study, we disentangled genome dimerization from other steps of the viral life-cycle and comprehensively mapped structure determinants of HIV-1 genome dimerization using a novel highthroughput approach that we call Functional Analysis of RNA Structure (FARS-seq). Using FARSseq, we found nucleotides throughout the HIV-1 5'UTR influencing dimerization and identified distinct structural conformations in monomeric and dimeric RNA. The dimeric RNA folded into a 'canonical' structure of the 5'UTR that displayed TAR, PolyA, PBS, and SL1-SL3 as stem-loops, and contained a long range U5-AUG interaction. In monomeric RNA, SL1 formed interactions with polyA and PBS. The PBS-SL1 interaction functionally couples primer binding with dimerization and the polyA-SL1 long-range interaction disrupts the major packaging motifs for Pr55Gag ${ }^{32-34}$. All in all, our data provide a mechanistic explanation for how RNA dimerization can be a prerequisite for packaging.

\section{Results}

Functional and structural analysis of RNA dimerization. HIV-1 genome dimerization largely depends on the stem of SL1 and its GC-rich palindromic loop sequence. Nevertheless, evidence suggests that RNA sequences and structures outside of SL1 also play a role therefore devised a strategy to exhaustively survey the 5'UTR for nucleotides influencing dimerization whilst at the same time generating information about RNA structure. We call this approach the Functional Analysis of RNA Structure (FARS-seq) (Fig. 1c). Fundamentally, FARSseq uses mutational interference to generate complete, unbiased, quantitative profiles of RNA function at single nucleotide resolution ${ }^{38,39}$ (Fig. 1d). These functional profiles are generated by 
physical separation of mutant RNA populations according to functionality followed by next generation sequencing and the analysis of mutation frequencies in the 'functional' and 'nonfunctional' populations. Simultaneously, structural profiles are obtained by treating the fractions with dimethyl sulfate (DMS), which is a chemical widely used for probing RNA structure ${ }^{40,41}$. DMS reacts with unpaired adenosine and cytosine bases to form adducts that can be read out as mutations on next generation sequencing machines ${ }^{42-44}$. Normally, DMS only provides information on whether a nucleotide is base paired, and not the identity of the base paring partner. However, when DMS modification is performed on mutational libraries it enables the direct detection of RNA stems (Fig. 1f) $)^{45,46}$. That is, when a mutation in the library occurs within a stem, it creates an unpaired nucleotide at the position facing the mutation. This newly unpaired residue becomes more accessible for DMS modification leading to correlated mutations in the sequencing data. Thus, FARS-seq combines two different mutational read outs to experimentally couple RNA structural and functional information.

To physically separate mutants according to their effects on dimerization, we took advantage of the observation that RNA transcripts containing dimerization signals are able to spontaneously associate in vitro, producing a dimeric RNA species that can be physically separated from the monomeric species on native agarose gels (Fig. 2a). Similar gel-based assays have been instrumental in the discovery of dimerization motifs in HIV-1 ${ }^{17-19}$ and other viruses ${ }^{47-49}$. This setup also disentangles the effect of RNA structure on dimerization from other factors, such as the binding of protein or other co-factors. To assess the effect of transcription start site heterogeneity on the dimerization properties of the HIV-1 genome we tested three transcript variants beginning with $1 \mathrm{G}$, $2 \mathrm{G}$ or $3 \mathrm{G}^{13,50}$ (Fig. 2a). For each of these transcript variants, we also tested whether capping affected dimerization and assessed their dimerization properties under low salt and high salt buffers favoring monomerization and dimerization, respectively (Fig. 2a). After physical separation on a native gel, bands corresponding to monomeric and dimeric RNA populations were excised and either left untreated or soaked in DMS. For the DMS sample (and its control), RNA was reverse transcribed in the presence of $\mathrm{Mn}^{2+}$ to allow mutagenic bypass of the modified nucleotides by the reverse transcriptase $\mathrm{e}^{44,51}$. In the absence of DMS, mutation frequencies in the mutated and non- 
mutated control library were $5.4 \times 10^{-3}$ and $3.7 \times 10^{-4}$, respectively, and the mutational interference libraries with a signal to noise $D_{m}(i)>2$ (Sup. Fig. 1; details in Sup. Methods). In the DMS treated samples, we saw an additional increase in mutation frequencies at the expected $A$ and $C$ residues indicating a successful modification of RNA (3.4- and 7.8-fold increase at C and A, respectively, Sup. Fig. 2).

RNA dimerization depends not only on SL1, but also on other regions within the HIV 5'UTR. We first asked which regions of the RNA were required for dimerization using mutational interference mapping (MIME) to calculate $\mathrm{K}^{\text {dimer }}$ values for each nucleotide position. This metric is related to the ratio of mutation frequencies in the monomer vs dimer RNA. For computational analysis however, these ratios are corrected for errors introduced during library preparation and sequencing (mechanistic derivation in Sup. Methods). Thus, $\mathrm{K}^{\text {dimer }}$ is a quantitative measure of the relative effect of each mutation on dimerization. Across all samples and conditions, median $\log _{2}\left(K^{\text {dimer }}\right)$ values were heavily skewed towards positive values indicating that most mutations inhibited, rather than enhanced, dimerization indicating that the HIV-1 genome is highly optimized to dimerize as a key part of its life cycle (Fig. 2b). By segregating $K^{\text {dimer }}$ values by structural domain we found that most dimerization inhibiting mutations mapped to SL1 (Fig. 2b). Although less prominent than SL1, many other domains exhibited skewed distributions. Mutations to SL3, SL4, and polyA were biased towards inhibiting dimerization whereas mutations to TAR and SL2 preferentially enhanced dimerization. In contrast, mutations to the inter-domain regions were largely neutral with a narrow distribution centered around zero (Fig. 2b).

We next plotted median $\log _{2}\left(\mathrm{~K}^{\text {dimer }}\right)$ values at each nucleotide position for capped and uncapped transcript variants measured under the two buffer conditions (Fig. 2c, 2d; Sup. Fig. 3). All conditions exhibited a very large peak that localized to SL1, as well as a smaller double peak mapping to SL3 (Fig. 2c, 2d). In high salt buffer most mutations inhibited dimerization, whereas under low salt conditions it was possible to distinguish additional dimerization enhancing or inhibiting regions (Fig. 2c, 2d). Notably, sequences surrounding the AUG start codon and mapping to U5 were both required for dimerization in low salt buffer, suggestive of a functionally important U5-AUG interaction (Fig. 2d). A double peak also emerged within the region 122-141 in low salt 
buffer (Fig. 2d). This region contains the primer activation sequence (PAS) which hints that structural changes in the PBS domain may regulate RNA dimerization ${ }^{52,53}$. Conversely, we found regions within TAR, polyA, PBS and SL2 that enhanced dimerization upon mutation (Fig. 2d). The strongest of these regions mapped to the 3' end of PBS and SL2. Taken together, these data reinforce the key importance of SL1 for genome dimerization, but also reveal sequences outside of SL1 participate in the dimerization process.

$1 G$ and 3G RNAs have different dimerization properties. Because the HIV-1 transcription start site has been reported to alter the structure of the HIV-1 5'UTR, we next tested which RNA sequences were important for dimerization within the 1G, 2G, and 3G uncapped variants (Fig. 2ef, Sup. Fig. 4). We did this by plotting the absolute difference between the median $\log _{2}\left(K^{\text {dimer }}\right)$ values of each variant to the mean values of the three transcripts. In high salt buffer, most positions were unchanged in the $1 G, 2 G$ and $3 G$ variants (less than $\Delta 0.25 \log _{2}\left(K^{\text {dimer }}\right)$ variant - mean) (Sup.

Fig. 4, Fig. 2e). The only exception were nucleotides mapping to the SL1, which were functionally more important in the $3 G$ variant, and less important in the $1 G$ variant. Upon performing a similar analysis for the low salt condition, distinct functional profiles for the $1 \mathrm{G}$ and $3 \mathrm{G}$ transcript variants emerged, with divergence across regions compared to the mean of the three transcripts (Sup. Fig

4, Fig. 2f). The $3 G$ variant had increased dependence on a region spanning the U5 and PAS (nts 105-117 and nts 125-131) and sequences surrounding the AUG start site (nts 335-344). Increased dependencies of smaller magnitudes were also observed in the tRNA primer binding site (nts 182200), the anti-PAS (nts 217-223), regions flanking SL1 such as the CU rich motif (nts 228-247), a region in SL2 (nts 299-300), and a G rich region downstream of the AUG start codon (nts 360-366). We note that the regions in TAR, PBS, and SL2 that enhanced dimerization upon mutation in low salt conditions behaved identically in $1 G, 2 \mathrm{G}$, and $3 \mathrm{G}$ variants, meaning that they impact

157 dimerization in a way that is unrelated to transcription start site selection. We also remarked that the $1 \mathrm{G}$ and $2 \mathrm{G}$ transcripts variants behaved similar in both buffer conditions (Sup. Fig. 4), with a reduced dependency on regions external to SL1 for dimerization. Our interpretation is that the $1 \mathrm{G}$ and $2 \mathrm{G}$ transcripts readily fold into a dimer promoting conformation, whereas the $3 G$ variant has a reduced capacity to dimerize. Capped and uncapped transcripts had near identical functional 
profiles (Sup. Fig. 3). The only region that differed mapped to polyA, providing indirect evidence of a functional interaction between the 5' cap structure and polyA (Sup. Fig. 4).

Distinct structural signals in monomeric and dimeric RNA. So far, the analysis of the functional profiles demonstrate that sequences involved in genome dimerization map to distinct regions of the HIV-1 5'UTR. These sequences may fold into RNA structures that are necessary for genome dimerization itself, or indirectly regulate genome dimerization by altering folding pathways. We therefore next determined RNA structural motifs present in monomers and dimers by analyzing the DMS reactivities of the FAR-seq data.

As before, we analyzed capped and uncapped 1G, 2G, 3G transcript variants in both monomer and dimer buffers. Correlations between DMS reactivities at each position amongst all conditions were very high (Fig. 3a; Kendall rank correlation coefficients, mean 0.84, min 0.70, max 1.0) suggesting that significant portion of the 5'UTR was folded into a similar conformation under all conditions. Nevertheless, hierarchical clustering of the DMS reactivities revealed a clear structural distinction between monomer and dimer, as well as between the $1 G / 2 G$ and $3 G$ transcript variants (Fig. 3a). In contrast to the functional profiling, where buffer conditions had a very large effect on the functional profiles, structural information obtained under both conditions were highly correlated (correlation coefficients; low salt 0.84 , high salt 0.85 ), as were uncapped and capped RNAs (correlation coefficients; capped 0.85, uncapped 0.84). The first branchpoint separated 1G/2G dimer structures from the $1 \mathrm{G} / 2 \mathrm{G}$ monomer and $3 \mathrm{G}$ structures. Subsequent branching grouped $1 G / 2 G$ monomer structures away from the $3 G$ structures. Finally, $3 G$ structures separated into monomer and dimer subclusters. These four structural groupings were also supported by principal component analysis (PCA) of DMS reactivities, which separated monomer from dimer, and $3 G$ variants from $1 \mathrm{G} / 2 \mathrm{G}$ variants (Fig. 3b). Guided by the PCA and hierarchical clustering, we pooled DMS reactivity data into 4 structural groups: $3 G$ dimer, $3 G$ monomer, $1 G / 2 G$ monomer, $1 G / 2 G$ dimer. Interestingly, across all samples, variance in DMS reactivities localized mainly to polyA and SL1 (Fig. 3c). To further explore this, we used a statistical approach to compare DMS reactivities in the $1 G / 2 G$ dimer cluster with the $3 G$ monomer cluster as these were the most structurally divergent samples (correlation coefficient 0.740) (Fig. 3d). Between these clusters, we found 
statistically significant changes in reactivity that again remained localized to polyA and SL1 (Fig. 3d and Sup. Files).

To obtain information on RNA secondary structure differences between these structural classes we used pooled DMS reactivities as soft constraints to guide in silico RNA folding ${ }^{54,55}$ (Fig. 4, Sup. Fig 5 and Sup. Fig 6). For the 1G/2G dimer class we obtained an RNA structure that closely resembled the 'canonical' HIV-1 5'UTR (Fig. 4a and Sup. Fig 6). This structure contains the TAR, PolyA, PBS, SL1 and SL3 stem loops, as well as the AUG-U5 interaction. The basal portion of SL1 folded into an extended form containing unpaired purines that are important for genome packaging ${ }^{32,56}$. SL2, which can fold into alternative stem loop structures, folded as an imperfect stem-loop that exposes part of the U1snRNA binding site within the loop, and SL3 folded into its canonical short stem loop structure. We then assessed the robustness of this prediction by computing Shannon entropies of base pairing probabilities at each position in the 5'UTR (Fig. $\mathbf{4 b}$, Sup. Fig. 5). Low entropy values throughout the 5'UTR indicated high confidence in the prediction and a well-ordered structure with only some ambiguity in the base pairing at the basal portion of SL1. This was confirmed by dot plots of base pairing probabilities and a bootstrapping analysis showing high confidence stem loop structures for the TAR, PolyA, PBS, SL1 and SL3 stem loops, as well as the AUG/U5 interaction (Fig. 4c and Sup. Fig. 5).

We next analyzed the structure of the $3 G$ monomer sample, finding that it was dramatically reorganized (Fig. 4d). The most striking changes were seen in the polyA, AUG-U5, and SL1. PolyA and SL1 no longer folded into their canonical stem-loops. Instead, these stem loops were reorganized into a long-distance interaction, with the GCGCGC palindromic loop of SL1 base pairing with the apical portion of the polyA stem. The AUG-U5 interaction was also no longer present; U5 now base paired with the 5' stem of SL1, and the AUG containing region fold into a stem loop structure also referred to as SL4. Finally, we observed a new long-distance interaction between polyA and a region within the Gag coding sequence (nts 358-367). The SL1-PolyA reorganization was well supported by the DMS reactivity changes (Fig. 4 and Sup. Fig. 6). In particular, the unpaired adenosine $263 \mathrm{~A}$ in the SL1 loop, which was highly reactive in the dimer structure, became unreactive in the monomer due to base pairing with U87. Similarly, nucleotides 
C84 and C85 in polyA, which were reactive in the dimer structure, became unreactive in the monomer due to base pairing with $265 \mathrm{G}$ and $266 \mathrm{G}$ in the SL1 stem. Finally, A89 in the stem of polyA, which was unreactive in the dimer structure, became unpaired in monomer structure and reactive to DMS. Shannon entropies, base paring and bootstrapping probabilities at the predicted PolyA-SL1 interaction indicated some uncertainty in the prediction, especially within U5 and the 5 ' portion of SL1 (Fig. 4 f and Sup. Fig 5). Remarkably, despite the dramatic reorganization of PolyA and SL1, a large proportion of the 5'UTR folded identically in $1 \mathrm{G} / 2 \mathrm{G}$ dimer and $3 \mathrm{G}$ monomeric populations, with PBS, SL2 and SL3 unchanged. Interestingly, TAR was present in all predictions, but in the $3 G$ monomer the first nucleotides in the base of TAR became single stranded, and 227 potentially more available for the translation machinery. a stem-loop and the $1 \mathrm{G} / 2 \mathrm{G}$ monomer population folded into the alternative polyA-SL1 containing 230 structure (Sup. Fig. 5). However, these two structural classes showed increased Shannon entropies in polyA, U5, SL1 and the Gag coding sequence when compared to the 1/2G dimer and $3 G$ monomer structures. Thus, $3 G$ dimer and $1 / 2 G$ monomer populations are structurally less uniform, even though we selected for pure dimer and monomer structures in the native gels. The most likely explanation is that these structures partially return to equilibrium after isolation, probably during the probing reaction at $37^{\circ} \mathrm{C}$.

Altogether, these data support a novel structural rearrangement of the HIV 5'UTR leading to extensive base pairing between SL1 and the polyA-U5 region. This monomeric rearrangement appears to be favored in the $3 G$ populations, whereas the $1 \mathrm{G} / 2 \mathrm{G}$ population tend towards the dimer structure.

Two-dimensional RNA structural probing detects structural differences in monomer and dimer populations. The incorporation of information from RNA structural probing experiments improves the accuracy of RNA structure predictions, but structural elements can still be incorrectly predicted because data from chemical probing experiments typically provide information on whether a nucleotide is base-pair or not, but not its base pairing partner ${ }^{57,58}$. FARS-seq enables a 
more powerful model-free approach to RNA structure determination by exploiting information in the mutation library to identify RNA helices directly (Fig. 5a). When mutating a nucleotide in a stem structure, the base pairing partner, now unpaired, becomes more reactive to the chemical probe 248 leading to correlated mutations in the sequencing data ${ }^{46,58}$. This two-dimensional data can directly detect RNA helices (along the diagonal) as well as non-canonical and tertiary interactions that are otherwise impossible to predict from classical one-dimensional RNA structural probing experiments.

Signals for RNA helices were visible in the raw mutational and z-score normalized data along the diagonals (Fig. 5b, 5e and Sup. Files). These signals were refined by applying convolution and threshold filters to enhance stems as well as tertiary interactions (Fig. 5b and $\mathbf{5 e}$ ). Finally, high confidence stems were highlighted by applying a helix filter and algorithm to select the 'best' nonconflicting stems with the highest score (Fig. $\mathbf{5 c}$ and $\mathbf{5 f}$ ). Stem signals corresponding to SL1 were systematically present in dimer selected samples and absent in monomer selected samples (Sup. Fig 8 and Sup. Files). In the 1G/2G dimer sample, both SL1 and polyA stem signals were observed. In the $3 \mathrm{G}$ monomer, polyA and SL1 stems were replaced with a signal matching the long distance SL1-polyA interaction (compare Fig. $\mathbf{5 b}$ and $\mathbf{5 c}$ with $\mathbf{5 e}$ and $\mathbf{5 f}$ ). Unexpectedly, in the $3 \mathrm{G}$ monomer we detected an additional novel interaction between the PBS loop and SL1, as well as a weaker signal between TAR and PBS, both of which were supported by a bootstrapping analysis (Fig. 4e, 4f and Sup. Fig. 8). In the previous structural prediction these regions in PBS and SL1 had high Shannon entropies and were poorly resolved (Fig. 4).

Intriguingly, and uniquely in the $1 \mathrm{G} / 2 \mathrm{G}$ structures, the TAR and polyA stem signals in the filtered z-scores were accompanied by punctate signals characteristic of tertiary contacts, alternative folds or non-canonical base-pairings (Sup. Fig. 6). Because these contacts were consistently present in the $1 G / 2 G$ samples and missing from the $3 G$ samples, we speculate that they help to stabilize the 5' end of the HIV-1 transcript to inhibit the translation of 1G/2G transcripts (Sup. Fig. 7 and Sup.

270 Files) $)^{13}$. Additionally, in the $1 \mathrm{G} / 2 \mathrm{G}$ monomer, the mutually exclusive polyA stem and the polyA271 SL1 interaction were both observed, strengthening the idea that $1 \mathrm{G} / 2 \mathrm{G}$ samples are preferentially dimeric and that some interconversion occurs even when monomers are isolated (Sup. Fig. 7). 
273 To obtain enhanced structural models of the dimer and monomer structures we focused on the $2741 G / 2 G$ dimer and $3 G$ monomer samples as these were the most structurally uniform. Here, the 275 best stems obtained by multidimensional chemical probing were used as additional hard 276 constraints in RNA structure prediction (Fig. $\mathbf{5 d}$ and $\mathbf{5 g}$ ). The enhanced $1 \mathrm{G} / 2 \mathrm{G}$ dimer structure was 277 nearly identical to that obtained without hard constraints, and contained the TAR, PolyA, PBS, SL1 278 and SL3 stem loops, as well as the AUG/U5 interaction as previously predicted (Fig. 5d). The 279 enhanced 3G monomer structure contained TAR, polyA-SL1 interaction, SL2, SL3 SL4 and polyA280 Gag interaction, as before, but now included a stem loop structure due to base paring between PBS and SL1 (Fig. 5g). A TAR-PBS pseudoknot interaction was added post-hoc, as it was selected 282 by the best stem algorithm and supported by a bootstrapping analysis, although we note that the $2 \mathrm{~d}$ stem score was relatively weak. All in all, multidimensional chemical probing not only provided direct experimental evidence that $1 \mathrm{G} / 2 \mathrm{G}$ dimer and $3 \mathrm{G}$ monomer fractions are structurally distinct, but also identified structural features that couldn't be predicted by classical RNA structural probing 286 experiments.

SL1 stability is a key element for genome dimerization. One of the strengths of FARS-seq is the coupling of RNA structural and functional information at single nucleotide resolution. We therefore mapped the $\mathrm{K}^{\text {dimer }}$ values onto the dimer and monomer structures. In both buffers, the median mutations with the strongest effects mapped to the apical portion of SL1, with mutations to the palindromic loop sequence revealed to be the most destabilizing for dimerization, in agreement with their crucial role in the kissing loop interaction (Fig. 6a, see Extended $\mathbf{K}^{\text {dimer }}$ Data Tables). The unpaired adenosine residues flanking the loop sequence were less important for dimerization than the palindromic sequences, in keeping with the observation that they can be individually mutated without disrupting dimerization ${ }^{59}$. Mutations to the stem of SL1 also strongly inhibited dimerization, with apical stem mutations generally having a stronger effect on dimerization compared to the basal stem mutants $\left[\log _{2}\left(K^{\text {dimer}}\right)\right.$ values $0.61-6.85$ vs $\left.0.31-3.49\right]$ (Sup. Fig. 7 ).

298 Surprisingly, mutations at several positions within the SL1 internal loop (G247, A271, G272, G273) strongly enhanced dimerization upon mutation (Fig. 6a and 6b, Sup. Fig. 7, Extended K ${ }^{\text {dimer }}$ Data

Tables). Dimer enhancing mutations at these positions presumably stabilize SL1 by closing or 
reducing the size of the internal loop, strongly indicating that SL1 stability is a critical parameter for 302 dimerization.

303 Whilst two-dimensional structural probing identified SL1 as a short stem loop with an apical and 304 basal stem separated by an internal loop (nucleotides 243-277), our data nevertheless reveals 305 structural plasticity in SL1. This realization comes from mapping the functional data to different 306 extended forms of SL1 that have been proposed in the literature: a two-internal loop model (2IL), a 307 three-internal loop model (3IL), and three-way junction (3WJ) model (Fig. 6b). Even though these 308 models have mutually exclusive internal loop configurations, mutations that closed or reduced the 309 size of SL1 internal loops were invariably dimerization enhancing (Fig. 6b, green arrows). For 310 example, A235C, A235U or G281U strongly enhanced dimerization by converting the A235-G281 311 internal loop into a base pair in the three-way junction (3WJ) model, even though these mutations 312 would have no effect on SL1 stability on the other structural models (Fig. 6b, green arrows). 313 Similarly, G282C and G239C would close the internal loop in the 3 internal loop (3IL) model 314 explaining their dimerization enhancing properties (Fig. 6b, green arrows). To confirm the structural 315 plasticity of SL1, we performed in solution DMS-MaPseq analysis of mutants A235C and A239C 316 and showed that they reconfigured the SL1 stem, as predicted (Sup. Fig. 9). Interestingly, 317 mutations $\mathrm{A} 242 \mathrm{C}$ or $\mathrm{A} 242 \mathrm{U}$ reduced the size of an SL1 internal loop in all models but nevertheless 318 disrupted dimerization (Fig. 6b and 6c; red arrows). These functional effects are explained by the 319 fact that A242C or A242U extend the PBS-SL1 interaction to stabilize the monomer structure. Thus, 320 the core dimerization structure in SL1 comprises an apical $7 \mathrm{nt}$ stem and a basal $4 \mathrm{nt}$ stem 321 separated by an internal loop that can be further stabilized by metastable stem extensions or 322 disrupted by a base-pairing interaction with PBS.

323 Inter-domain interactions regulate dimerization. Outside of SL1, we found several structural 324 domains and inter-domain interactions that affected dimerization (Fig. 6). Our data support a role 325 for the AUG-U5 interaction in positively regulating dimerization, as conversion of GU base pairs at 326 U107-G342, G108-U341, G112-U337 to either AU or GC base pairs consistently enhanced 327 dimerization, whereas mutations disrupting the interaction were inhibitory (Fig. 6a). SL3 stem 328 mutations weakly inhibited dimerization, most likely because disruption of SL3 would induce 
misfolding of the RNA (Fig. 6a). Finally, mutations to SL2 were generally dimerization enhancing, and these types of mutations were especially evident in the 3' SL2 stem (Fig. 6a).

We also validated the novel short- and long-range interactions between polyA-SL1 and PBS-SL1. Mutations to the base of polyA generally inhibited dimerization, indicating that destabilizing the polyA stem favours the formation of the polyA-SL1 interaction (Fig. 6a). On the other hand, mutations to the upper portion of polyA enhanced dimerization by disrupting the polyA-SL1 base pairing (Fig. 6c). In the same vein, we found stretches of nucleotides in PBS that strongly enhanced dimerization upon mutation (Fig. 6c). Functional profiles in the lower PBS stem were particularly interesting as this stem structure is universally found in contemporary models of the HIV-1 5'UTR and contains the primer activation sequence (PAS) known to be important for efficient reverse transcription $^{60}$. We found that mutation of two nucleotides G217 and C218 in the lower PBS stem very strongly enhanced dimerization, even though mutations to this stem were generally inhibitory (Fig. 6a). This can be mechanistically explained because mutation of these nucleotides disrupted a novel base-pairing between PBS and SL1 that stabilizes the monomer structure.

Because these results suggested a functional interaction between primer tRNA binding and dimerization, we next assessed whether disruption of the PBS with tRNA mimic oligos affected dimerization. CPBS $182-199$ annealed to the loop region disrupted the putative TAR-PBS interaction, whereas cPBS199-216 disrupted the novel PBS-SL1 stem loop (Fig. 7a). Both oligos enhanced dimerization confirming a functional interaction between PBS and dimerization. Surprisingly, annealing the $\mathrm{CPBS}_{182-199}$ oligo also led to the formation of a higher, presumably tetrameric molecular species. The TAR apical loop contains a 10-nucleotide palindromic sequence that has been proposed to dimerize by a TAR-TAR kissing interaction analogous to the one used by SL126. We therefore postulate that CPBS $_{182-199}$ disrupts the TAR-PBS interaction detected by multidimensional structural probing, allowing TAR to dimerize independently of SL1.

Finally, since genome dimerization is thought to be a pre-requisite for genome packaging, we selected mutations in adjacent nucleotides with divergent effects on dimerization and measured their effects on Pr55 ${ }^{\text {Gag }}$ binding by microscale thermophoresis (MST) (Fig. 7b). Importantly, none 
of these mutations resided in the HIV-1 packaging domain (SL1-SL3). In PBS, C218G, which strongly enhanced dimerization had higher affinity $\left(K_{d} 19 \mathrm{nM}\right)$ to Pr55 ${ }^{\mathrm{Gag}}$ compared with WT RNA (Kd $38 \mathrm{nM})$. In contrast, PBS A220G-G221A, which was unable to dimerize, did not bind Pr55 ${ }^{\mathrm{Gag}}$ at any of the concentrations tested ( $K_{d}$ n.a.). In polyA, dimerization enhancing mutation C84A-C85C bound Pr55 Gag with higher affinity (17 nM) than WT, whereas dimerization disrupting mutation U86G-A89C bound Pr55 ${ }^{\text {Gag }}$ with lower affinity than WT (110 nM). In solution DMS-MaPseq analysis of these four mutants validated their effects on the polyA-SL1 and PBS-SL1 interactions (Sup. Fig 10). We also introduced these mutations into the full-length HIV-1 genome and assessed their effects on packaging efficiency in competition assays (Fig. 7c). In PBS, dimer promoting mutant C218G was enriched 1.5 -fold in virions compared to the monomer promoting mutant A220GG221A. In polyA, dimer promoting mutant C84A-C85A was enriched 2-fold in virions compared to the monomer promoting mutant U86G-A89C. In a five-way competition assay between wild-type (WT) HIV-1 and the mutants, dimer promoting mutants C218G and C84A-C85A were packaged equivalently or better than WT. Conversely, monomer promoting mutants U86G-A89C and A220GG221A were deficient in packaging compared to WT. In cell DMS-MaPseq analysis of these four 371 mutants again validated their effects on the polyA-SL1 and PBS-SL1 interactions (Sup. Fig 11).

372 Together, these results provide a clear mechanistic explanation for the link between dimerization, 373 Pr55 $5^{\text {Gag }}$ binding and packaging. Furthermore, we show how changes to the PBS functionally link 374 the tRNA binding region to packaging (Fig. 7 d).

\section{Discussion}

376 Accumulating evidence emphasizes dimerization as a key step in HIV-1 life cycle that is regulated, 377 at least in part, through the folding of the HIV-1 genomic RNA ${ }^{5,11,62,12-16,27,28,61}$. Here, we resolved 378 the structure of the monomeric and dimeric RNAs using a novel approach that integrates 379 information from RNA structural probing with high-throughput functional profiling. This experimental strategy has significant advantages over other chemical probing methods that make ensemble measurements over all possible conformations of the RNA in solution. Such ensemble

382 measurements, unless cautiously interpreted, can lead to false predictions when mapped to a 

with respect to their function, akin to in gel SHAPE which was first developed to resolve structural differences between monomeric and dimeric species of the HIV-1 5'UTR ${ }^{28}$. Moreover, by performing chemical probing on mutagenic libraries we obtain model-free information on RNA helices in the same way as "mutate and map"58 or "M2-seq"46. Finally, and most importantly, our approach enables a deep understanding of how RNA structures relate to RNA function by uniquely coupling structural information with a functional read-out.

Taken together, our data recognizes a core dimerization domain of SL1 comprised of a 7 bp apical stem and 4 bp basal stem separated by an internal loop. This core dimerization domain is present in most structural models of SL1, but there is significant disagreement on whether SL1 is further extended $^{63-66}$. In some structures, extensions to SL1 even lead to the complete disruption of SL2 ${ }^{12}$. Here, we found no direct evidence that SL1 is in an extended form in dimeric RNA and consistently observe signals for SL2 as a short imperfect stem containing a bulged adenosine. Nevertheless, functional profiling provides strong evidence that mutually exclusive extended forms of SL1 can be readily generated, either directly through stabilizing mutations, or indirectly by destabilizing SL2. The fact that single point mutations could have such dramatic effects on dimerization provides evidence that the 5'UTR is dynamic and metastable. In the context of viral infection this is noteworthy because it provides a mechanism to regulate dimerization through the binding of viral or cellular factors to the genome (Fig. 7c).

The metastable nature of SL1 was strikingly revealed in monomeric RNA. In contrast to SL3, which was present in both monomer and dimer structures, SL1 was destructured in monomeric RNA. Instead of a stem-loop, SL1 was reorganized into a short-range interaction with PBS and a longdistance interaction with polyA. These results are in agreement with the prevalent idea that RNA conformational switches regulate HIV-1 replication ${ }^{33,67}$. The dimer and monomer structural conformations we present here are reminiscent of the branched multiple hairpin $(\mathrm{BMH})$ and longdistance interaction (LDI) models that were proposed as alternative structures that would regulate the dimerization, packaging, splicing and translation of the HIV-1 genome $e^{5,15,16,27}$. The $\mathrm{BMH}$ exposes the TAR, polyA, PBS, SL1, SL2 and SL3 structures, and contains the U5-AUG interaction. 
411 The LDI model includes the interaction between polyA and SL1, but also includes additional 412 rearrangements that we did not observe, such as an extension of SL3 and a disruption of SL2. 413 Moreover, the LDI model does not include the novel PBS-SL1 interaction. Nevertheless, certain 414 mutants designed to alter the LDI/BHM equilibrium are directly applicable to our structural model. 415 In particular, mutations destabilizing the polyA stem inhibit dimerization and packaging ${ }^{15,27}$, whereas mutations disrupting the polyA-SL1 interaction enhanced dimerization ${ }^{16}$. These data are 417 in agreement with our results showing that polyA-SL1 regulates not only dimerization, but also genome packaging. Significantly, recent work has identified the primary Pr55 ${ }^{\text {Gag }}$ binding site for HIV-1 as SL1 $1^{32,38,39,68}$ with polyA providing an additional packaging signal in cells ${ }^{39,69}$. The fact that SL1 and polyA are completely disrupted in the monomer population provides a mechanistic explanation for the long-postulated link between dimerization and packaging.

Recently the structure of the $3 G$ capped transcript was solved by NMR revealing the disruption of the polyA stem in $3 G$ transcripts and the formation of a long range interaction between SL1 and $U 5^{14}$. Thus, our results agree that $3 G$ transcripts are preferentially monomeric, yet disagree with precise structural details, in particular the base pairing partner of SL1. One way to reconcile these data is that the NMR structure was obtained with the Mal isolate, in contrast to the NL43 isolate used in the present study. Mal is a complex recombinant containing a 23-nucleotide duplication in the PBS, strikingly in the same region regulating dimerization in this study. Moreover, this PBS duplication in Mal leads to marked structural differences in the initiation of reverse transcription compared to the prototypic subtype B strain NL43 ${ }^{52,53}$. It is therefore plausible that Mal and NL43 isolates use related, yet distinct, structural rearrangements to regulate dimerization. Nonetheless, both the polyA-SL1 and PBS-SL1 interactions are conserved amongst 800 curated sequences in

433 the Los Alamos HIV-1 sequence database indicating regulation of dimerization by polyA and PBS 434 is widespread (Sup. Files).

435 Finally, we identified a novel interaction between PBS and SL1 that acts as negative regulator of 436 dimerization, $\operatorname{Pr} 55^{\mathrm{Gag}}$ binding and packaging. We demonstrated that this negative regulation can 437 be counteracted through the binding of oligos to the primer binding site. Disruption of this negative regulation would mechanistically explain why tRNA annealing enhances dimerization ${ }^{31,70}$, and also 
opens up the possibility that primer binding to the PBS affect other steps of the HIV-1 life-cycle, such as translation, by altering the monomer / dimer equilibrium. It also reveals a general principle by which RNA structural changes induced by host factors can regulate key stages of the HIV-1 life cycle (Fig. $\mathbf{7 d})$.

\section{Methods}

Plasmid. NL43 sequences were obtained from pDRNL43 $\Delta$ Env plasmid, which is containing fulllength NL43 but without flanking cellular sequences ${ }^{71}$ and contains a deletion in Env for biosafety.

Protein expression and purification. Expression, purification and characterization of NL4.3 Pr55 ${ }^{\mathrm{Gag}}$ with an appended C-terminal His6-tag was performed as described by McKinstry et al. ${ }^{72}$

Mutant library preparation. DNA templates were prepared by PCR using Taq DNA polymerase (NEB) with RNA expression plasmid pDRNL43- $\triangle$ Env and forward primers containing T7 RNA polymerase promoter and 3G/2G/1G at 5'end AAAgaagacTTggggTAATACGACTCACTATAGGGTCTCTCTGGTTAGACCAG / AAAgaagacTTggggTAATACGACTCACTATAGGTCTCTCTGGTTAGACCAG / AAAgaagacTTggggTAATACGACTCACTATAGTCTCTCTGGTTAGACCAG and reverse primer mGmATCTAAGTTCTTCTGATCCTGTCTG. PCR amplifications were performed in 1X reaction buffer, $0.2 \mathrm{mM}$ dNTPs, $250 \mathrm{nM}$ forward primer and reverse primer, $1 \mathrm{ng}$ plasmid as template, 1.25 U Taq DNA polymerase (NEB) using the PCR cycling conditions: $98^{\circ} \mathrm{C}$ for $30 \mathrm{~s}$, followed by 32 cycles of $98^{\circ} \mathrm{C}$ for $10 \mathrm{~s}, 60^{\circ} \mathrm{C}$ for $30 \mathrm{~s}$, and $68^{\circ} \mathrm{C}$ for $1 \mathrm{~min}$. Products were visualized by electrophoresis on 1\% agarose gels in 1X TAE buffer and column purified with NucleoSpin Gel and PCR Clean-up kit (Macherey-Nagel). The purified PCR products were used as template for error prone PCR using the Mutazyme II DNA polymerase (Agilent) and forward primer TAATACGACTCACTATA and reverse primer

\section{GTCTCGTGGGCTCGGAGATGTGTATAAGAGACAGGATCTAAGTTCTTCTGATCCTGTCTG.}

The PCR reaction volume was $50 \mu \mathrm{L}$ and consisted of $2 \mathrm{ng}$ of template DNA, $1 \mathrm{X}$ buffer, $200 \mu \mathrm{M}$ dNTPs, $0.25 \mathrm{mM}$ of each primer, $2.5 \mathrm{U}$ of Mutazyme II DNA polymerase. PCR cycling conditions 
were $95^{\circ} \mathrm{C}$ for $2 \mathrm{~min}$ followed by 35 cycles of $95^{\circ} \mathrm{C}$ for $30 \mathrm{~s}, 35-42^{\circ} \mathrm{C}$ for $30 \mathrm{~s}$ and $72^{\circ} \mathrm{C}$ for $1 \mathrm{~min}$. Products were visualized by electrophoresis on $1 \%$ agarose gels in $1 \mathrm{X}$ TAE buffer. A final column purification was carried out with the NucleoSpin Gel and PCR Clean-up kit (Macherey-Nagel) kit.

RNA preparation. Purified WT and mutated PCR products (900 ng) were used as templates for RNA in vitro transcription with a homemade T7 RNA polymerase. Reaction contained 1X reaction buffer (40 mM Tris pH 7.5, $18 \mathrm{mM} \mathrm{MgCl} 2,10 \mathrm{mM}$ DDT, $1 \mathrm{mM}$ Spermidine), $5 \mathrm{mM}$ NTPs, $40 \mathrm{U}$ RNasin (Molox), $900 \mathrm{ng}$ DNA template, and $0.05 \mathrm{U}$ of Pyrophosphatase, (NEB) and $5 \mu \mathrm{l}$ of homemade T7 RNA polymerase. The reaction was incubated at $37^{\circ} \mathrm{C}$ for $3 \mathrm{~h}$, followed by DNase I treatment for 30 min at $37^{\circ} \mathrm{C}$. RNA was gel purified after electrophoresis on $1 \%$ agarose gels in $1 \mathrm{X}$ TAE buffer using the NucleoSpin Gel and PCR Clean-up kit (Macherey-Nagel) with NTC buffer (Macherey-Nagel). Half of the purified RNA was capped with Vaccinia Capping System (NEB). Briefly, $10 \mu \mathrm{g}$ of RNA was mixed with nuclease-free $\mathrm{H}_{2} \mathrm{O}$ in a $1.5 \mathrm{ml}$ microfuge tube to a final volume of $15 \mu \mathrm{l}$. The sample was heated at $65^{\circ} \mathrm{C}$ for $5 \mathrm{~min}$, then placed on ice for $5 \mathrm{~min} .2 \mu \mathrm{l}$ of $10 \mathrm{x}$ capping buffer, $1 \mu \mathrm{l} 10 \mathrm{mM}$ GTP, $1 \mu \mathrm{l} 2 \mathrm{mM}$ SAM, and $1 \mu \mathrm{l}$ of Vaccinia Capping Enzyme were added and the sample was incubated at $37^{\circ} \mathrm{C}$ for 30 min. Capped RNA was column purified using the NucleoSpin Gel and PCR Clean-up kit (Macherey-Nagel) with NTC buffer (Macherey-Nagel).

Native agarose gel electrophoresis. RNA (600 ng) was denatured at $90^{\circ} \mathrm{C}$ for 2 min followed by chilling on ice for 2 min. RNA was incubated at $37^{\circ} \mathrm{C}$ for overnight $(15-17 \mathrm{~h})$ in high salt buffer (10 $\mathrm{mM} \mathrm{KH}_{2} \mathrm{PO}_{4}, \mathrm{pH} 7.4,1 \mathrm{mM} \mathrm{MgCl}, 122 \mathrm{mM} \mathrm{KCl}$ ) or low salt buffer (10 mM NaCl, $10 \mathrm{mM}$ Tris, $\mathrm{pH}$ 7.4). Samples were loaded with native loading dye (0.17\% Bromophenol Blue and $40 \%$ (vol/vol) sucrose) on 1\% agarose gel prepared with 1X Tris-Borate Magnesium (TBM) buffer (89 mM Tris base, $89 \mathrm{mM}$ boric acid and $0.2 \mathrm{mM} \mathrm{MgCl}$ ) and fractionated at $100 \mathrm{~V}$ for $85 \mathrm{~min}$ at room temperature. In some experiments, 12 pmol of oligos cPBS(182-199) GTCCCTGTTCGGGCGCCA and/or CPBS(199-216) TTCCCTTTCGCTTTCAAG were added to the RNA before denaturing to assess the effect of disrupting the PBS on dimerization.

Native polyacrylamide gel electrophoresis. RNA (800 ng) was denatured at $90^{\circ} \mathrm{C}$ for 2 min followed by chilling on ice for $2 \mathrm{~min}$. RNA was then incubated at $37^{\circ} \mathrm{C}$ for $30 \mathrm{~min}$ in high salt buffer 
492 (50 mM sodium cacodylate, $\mathrm{pH} 7.5,300 \mathrm{mM}, \mathrm{KCl}$, and $5 \mathrm{mM} \mathrm{MgCl}$ ) or low salt buffer (50 mM 493 sodium cacodylate, $\mathrm{pH} 7.5,40 \mathrm{mM} \mathrm{KCl}$, and $0.1 \mathrm{mM} \mathrm{MgCl} 2$ ). Samples were loaded with native 494 loading dye (0.17\% Bromophenol Blue and $40 \%$ (vol/vol) sucrose) on $4 \%$ acrylamide non495 denaturing gel prepared with 1X Tris-Borate Magnesium (TBM) (89 mM Tris base, $89 \mathrm{mM}$ boric

496

497

498

499

500

501

502

503

504

505

506

507

508

509

510

511

512

513

514 acid and $0.1 \mathrm{mM} \mathrm{MgCl} 2$ ) and fractionated at $150 \mathrm{~V}$ for $4 \mathrm{~h}$ at $4^{\circ} \mathrm{C}$, including 2 reference samples with SYBR gold (Invitrogen), which could be visualized under blue LED light. The dimer and monomer bands in samples were cut from the gel according to the position of reference samples by scalpel.

In gel DMS probing. Each gel piece from the polyacrylamide gel was divided into 2 parts. Half was soaked in $1 \mathrm{X}$ TBM containing $170 \mathrm{mM}$ DMS (dissolved in $\mathrm{EtOH}$ ), incubated at $37^{\circ} \mathrm{C}$ for $15 \mathrm{~min}$, followed by quenching with $50 \%$ (final) $\beta$-mercaptoethanol. The other half was soaked in $1 \mathrm{X}$ TBM ( $89 \mathrm{mM}$ Tris base, $89 \mathrm{mM}$ boric acid and $0.1 \mathrm{mM} \mathrm{MgCl}_{2}$ ) containing the equivalent volume of $\mathrm{EtOH}$ as the DMS treated sample, and incubated at $37^{\circ} \mathrm{C}$ for $15 \mathrm{~min}$. Gel slices were crushed into small pieces, soaked in 1X TBM ( $89 \mathrm{mM}$ Tris base, $89 \mathrm{mM}$ boric acid and $0.1 \mathrm{mM} \mathrm{MgCl}$ ) buffer at $4^{\circ} \mathrm{C}$ overnight. RNA was extracted using NucleoSpin Gel and PCR Clean-up kit (Macherey-Nagel) with NTC buffer (Macherey-Nagel).

Reverse transcription of $35 \mathrm{ng}$ of DMS modified RNA or $25 \mathrm{ng}$ of control RNA was performed with $200 \mathrm{U}$ SuperScript II reverse transcriptase (Invitrogen), $0.1 \mu \mathrm{M}$ of reverse transcription primer GTCTCGTGGGCTCGGAGATGTGTATAAGAGACAGGATCTAAGTTCTTCTGATCCTGTCTG, $0.5 \mathrm{mM}$ dNTPs, $50 \mathrm{mM}$ Tris $\cdot \mathrm{HCl}, \mathrm{pH}$ 8.0, $75 \mathrm{mM} \mathrm{KCl}, 6 \mathrm{mM} \mathrm{MnCl} 2,10 \mathrm{mM}$ DTT in $20 \mu \mathrm{l}$ reactions. The $R T$ reaction was incubated at $42^{\circ} \mathrm{C}$ for $3 \mathrm{~h}$.

Library preparation. For the functional probing MIME experiments, reverse transcribed cDNAs $\begin{array}{lllllll}\text { were } & \text { amplified } & \text { with } & 250 & \mathrm{nM} & \text { primers } & \mathrm{Fw} \text { - }\end{array}$

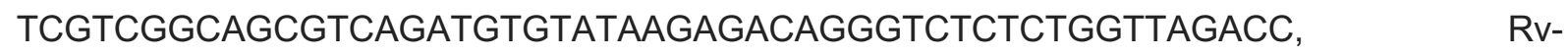
GTCTCGTGGGCTCGGAGATGTGTATAAGAGACAGGATGGTTGTAGCTGTCCCAG, $200 \mu \mathrm{M}$ dNTPs, 1X Q5 reaction buffer, Q5 polymerase (NEB) using the PCR cycling conditions: $98^{\circ} \mathrm{C}$ for $30 \mathrm{~s}$, followed by 32 cycles of $98^{\circ} \mathrm{C}$ for $10 \mathrm{~s}, 55^{\circ} \mathrm{C}$ for $30 \mathrm{~s}$, and $72^{\circ} \mathrm{C}$ for $30 \mathrm{~s}$. The PCR products 
were visualized by electrophoresis on $1 \%$ agarose gels in 1 X TAE buffer and column purified (using the NucleoSpin Gel and PCR Clean-up kit (Macherey-Nagel). 25 ng purified products were used in the final sequencing library preparation with Nextera DNA Flex Library Prep (Illumina) and Nextera DNA CD Indexes (96 Indexes, 96 Samples, Illumina), according to the manufacturer's instructions.

523 For structural profiling by DMS, we performed amplicon sequencing. PCR reaction volume was 25 $524 \mu \mathrm{l}, \quad 200 \quad \mu \mathrm{M} \quad \mathrm{dNTPs}, \quad 250 \quad \mathrm{nM} \quad$ primer $\quad$ pair 1 (Fw525 TCGTCGGCAGCGTCAGATGTGTATAAGAGACAGggtctctctggttagacc and RvGTCTCGTGGGCTCGGAGATGTGTATAAGAGACAGGCGTACTCACCAGTCGCC) or primer pair 2 (Fw-TCGTCGGCAGCGTCAGATGTGTATAAGAGACAGcgaaagtaaagccagaggag and Rv528 GTCTCGTGGgCtCGgAGATGtgtATAAGAGACAGCTCCCTGCTTGCCCATAC), 1X GXL reaction buffer, $0.625 \mathrm{U}$ of PrimeSTAR GXL DNA Polymerase (Takara Bio). Two PCR amplifications were performed using the PCR cycling conditions: $98^{\circ} \mathrm{C}$ for $30 \mathrm{~s}$, followed by 34 cycles of $98^{\circ} \mathrm{C}$ for $10 \mathrm{~s}, 60^{\circ} \mathrm{C}$ for $15 \mathrm{~s}$, and $68^{\circ} \mathrm{C}$ for $30 \mathrm{~s}$. Amplified libraries were column purified using the NucleoSpin Gel and PCR Clean-up kit (Macherey-Nagel). Paired end PE150 sequencing was carried out on an Illumina Novaseq instrument (Novogene).

Data analysis Sequencing data relating to MIME functional profiling experiments was first preprocessed using automated python scripts. First, sequencing reads were quality trimmed and stripped of adaptors with CutAdapt with the parameters "--nextseq-trim 35 - max-n 0 -A CTGTCTCTTATA -a CTGTCTCTTATA". Second, reads were aligned to the HIV-1 5'UTR using

538 Novoalign with the parameters "-o SAM -o SoftClip". Sam files were then analyzed using MIMEAnTo ${ }^{73}$ to generate Kdimer, which is a quantitative metric relating the effect of a mutation on dimerization (derivation in supplementary methods). Statistical methods used in MIMEAnTo are

541 described in detail elsewhere ${ }^{38,73}$.

542 Sequencing data relating to DMS structural probing was first preprocessed with ShapeMapper2 543 using parameters “--output-parsed-mutations --output-counted-mutations --render-mutation”. EtOH 544 treated and DMS treated raw sequencing reads were passed to ShapeMapper2 via the modified 545 and unmodified parameters, respectively ${ }^{51}$. DMS reactivities were calculated from ShapeMapper2 mutation rates using $90 \%$ Winsoring ${ }^{43}$. DMS reactivities were saved as XML files for processing 
547 with rf-fold module of the RNA Framework software package ${ }^{54,74}$. rf-fold was used to calculate 548 Shannon entropies and base pairing probabilities with the parameters "-ow -dp -KT -sh -g". Initial 549 RNA structure predictions of monomer and dimer conformations, using DMS reactivities as soft 550 constraints, were performed with rf-fold using the RNA folding algorithms in the Vienna RNA 2.0 551 package ${ }^{55}$. Refined RNA structure predictions using multidimensional probing results as additional 552 hard constraints were performed using RNAfold of the Vienna RNA 2.0 package ${ }^{55}$. Cluster maps 553 of DMS reactivities were generated using the clustermap function of the python Seaborn data 554 visualization library using 'kendall' correlation method and 'average' cluster method. Principal 555 Component Analysis was carried out using the PCA function of the python scikit-learn library. 556 Variances in DMS reactivities were calculated using the var function from the python NumPy library. 557 Pairwise comparison of DMS reactivities were carried out using a modified deltaSHAPE 558 calculation $^{75}$. This modified deltaSHAPE analysis uses several criteria to identify statistically 559 significant changes in reactivities. First, a z-factor test identifies nucleotides where DMS reactivities 560 change by $>1.96$ standard deviations of the DMS errors. Second, a standard score threshold of 1.5 is applied, meaning that delta reactivity values are at least 1.5 standard deviations away from the mean reactivity change. To filter these statistically significant sites for biological meaning, we next applied an absolute and a relative threshold filter. The absolute difference threshold ensures that a minimum reactivity change of 0.2 is needed for the site to be considered biologically relevant.

565 The relative threshold filter was set so that a relative change of at least 0.75 -fold was needed to 566 remove false positives where DMS reactivities are high in both conditions such that a large change 567 in reactivity is unlikely to affect RNA structure. RNA structures were visualized using Visualization 568 Applet for RNA (VARNA) ${ }^{76}$.

569 RNA structural interference by multi-dimensional structural probing was carried out using the M2570 seq pipeline ${ }^{46}$. Briefly, data was preprocessed by ShapeMapper into simple files which are string 571 representations of mutations in each read. Simple files were converted into the rich and compact 572 rdat format specific for RNA structure mapping experiments ${ }^{77}$. A two-dimensional matrix containing 573 mutation rates at pairs of nucleotide positions was constructed. Mutation counts were subsequently 574 normalized for total number of mutations along each row to give a true modification frequency. RNA 
structure signatures were further refined by calculating z-scores. A thresholding of 0 was applied to remove negative values, and a convolution filter was applied to enhance cross diagonal features. RNA helixes were finally identified in an unbiased manner by applying a filter for stems of Watson578 Crick and G-U wobble base pairs of at least 3 base-pairs in length. Best stems were predicted by eliminating conflicting stems through selecting the highest scoring stem. Bootstrapping analyses were performed using the rna_structure function of the Basic Inference Engine for RNA Structure (Biers) (https://ribokit.github.io/Biers/) using the default parameters (100 bootstrapping iterations).

582

583

584

585

586

587

588

589

590

591

592

593

594

595

596

597

598

599

600

601

Microscale Thermophoresis. RNA was labeled at the 3 ' end using pCp-Cy5 (Cytidine-5'phosphate-3'-(6-aminohexyl) phosphate) (Jena Biosciences) with T4 RNA ligase (NEB) overnight at $16^{\circ} \mathrm{C}$, followed by RNA Clean \& Concentrator Kits (ZYMO). $500 \mathrm{nM}$ labeled and purified RNA was denatured at $90^{\circ} \mathrm{C}$ for 2 min followed by chilling on ice for 2 min. RNA was folded at $37^{\circ} \mathrm{C}$ for overnight (15-17 h) in high salt buffer (10 mM KH $\left.2 \mathrm{PO}_{4}, \mathrm{pH} 7.4,1 \mathrm{mM} \mathrm{MgCl}_{2}, 122 \mathrm{mM} \mathrm{KCl}\right)$. For each binding experiment, RNA was diluted to $10 \mathrm{nM}$ in high salt buffer $\left(10 \mathrm{mM} \mathrm{KH}_{2} \mathrm{PO}_{4}, \mathrm{pH} 7.4,1\right.$ $\mathrm{mM} \mathrm{MgCl} 2,122 \mathrm{mM} \mathrm{KCl}, 0.01 \%$ Triton X-100, 10mM DTT and $0.02 \% \mathrm{BSA}$ ). A series of 16 tubes with $\operatorname{Pr} 55^{\text {Gag }}$ dilutions were prepared in high salt buffer, producing $\operatorname{Pr} 55^{\text {Gag }}$ ligand concentrations ranging from $30 \mathrm{pM}$ to $1 \mu \mathrm{M}$. For measurements, each ligand dilution was mixed with one volume of labeled RNA, which led to a final concentration of $5 \mathrm{nM}$ labeled RNA. The reaction was mixed by pipetting, incubated for $30 \mathrm{~min}$ at $37^{\circ} \mathrm{C}$, followed by $30 \mathrm{~min}$ on ice. Samples were then centrifuged at $10,000 \times \mathrm{g}$ for $5 \mathrm{~min}$. Capillary forces were used to load the samples into Monolith NT.115 Premium Capillaries (NanoTemper Technologies). Measurements were performed using a Monolith Pico instrument (NanoTemper Technologies) at an ambient temperature of $25^{\circ} \mathrm{C}$. Instrument parameters were adjusted to 5\% LED power, medium MST power, and MST on-time of 1.5 seconds. An initial fluorescence scan was performed across the capillaries to determine the sample quality and afterward, 16 subsequent thermophoresis measurements were performed. Data of three independently pipetted measurements were analyzed for the $\Delta$ Fnorm values, and binding affinities were determined by the MO. Affinity Analysis software (NanoTemper Technologies). Graphs were plotted using GraphPad Prism 8.4.3 software. 
602 In solution DMS-MaPseq (in vitro). RNA (300 nM) was denatured at $90^{\circ} \mathrm{C}$ for 2 min followed by 603 chilling on ice for 2 min. Next, RNA was refolded at $37^{\circ} \mathrm{C}$ for overnight (15-17 h) in high salt buffer 604 (10 mM KH2PO4, pH 7.4, 1 mM MgCl2, $122 \mathrm{mM} \mathrm{KCl).} \mathrm{DMS} \mathrm{was} \mathrm{added} \mathrm{to} \mathrm{the} \mathrm{RNA} \mathrm{solution} \mathrm{to} \mathrm{final}$ 605 concentration $170 \mathrm{mM}$, incubated at $37^{\circ} \mathrm{C}$ for $6 \mathrm{~min}$, followed by quenching with $\beta$-mercaptoethanol 606 and purification with ethanol precipitation. The purified DMS probed RNAs followed the same 607 reverse transcription and library preparation process as the in gel DMS probed RNA samples.

608 In solution DMS-MaPseq (in cells). 24hr prior transfection, $10^{7}$ HEK293T cells were plated in 10 $609 \mathrm{~mL}$ DMEM media containing 10\% FBS. 4 ug of plasmids expressing HIV-1 WT or HIV-1 mutants 610 were mixed with $48 \mathrm{uL}$ Polyethylenimine $(1 \mathrm{mg} / \mathrm{mL}$, Max 40k, Polysciences) and $500 \mathrm{uL}$ DMEM and 611 incubated for $10 \mathrm{~min}$ at room temperature before being added dropwise on the cells. $24 \mathrm{hr}$ post612 transfection the cells were probed by replacing the media with $3 \mathrm{~mL}$ DMEM containing $170 \mathrm{mM}$ 613 DMS and incubated at $37^{\circ} \mathrm{C}$ for 6 min. Cells were then washed with $5 \mathrm{~mL}$ PBS containing $140 \mathrm{mM}$ $614 \beta$-mercaptoethanol to quench the DMS. $1 \mathrm{~mL}$ TRI-reagent (Sigma-Aldrich) is directly added on the 615 cells to extract RNA according to the manufacturer's instructions. DNA contaminants were removed 616 by TurboDNase (Invitrogen) treatment for 30 min at $37^{\circ} \mathrm{C}$. RNA was purified using NucleoSpin Gel 617 and PCR Clean-up kit (Macherey-Nagel) with NTC buffer (Macherey-Nagel) and eluted in 20 uL of $6185 \mathrm{mM}$ Tris- $\mathrm{HCl} \mathrm{pH}$ 8.0. $7 \mathrm{uL}$ of the purified DMS probed RNAs were used for the reverse 619 transcription following the same reverse transcription and library preparation process as for the in gel DMS probed RNA samples.

621 Competition Assay. $24 \mathrm{hr}$ prior transfection, $7 * 10^{5}$ HEK293T cells were plated in $2 \mathrm{~mL}$ DMEM media containing 10\% FBS. For the co-transfection experiments, equal amount of plasmids expressing WT or mutants (600 ng total) were mixed with $7.2 \mathrm{uL}$ Polyethylenimine $(1 \mathrm{mg} / \mathrm{mL}$, Max 624 40k, Polysciences) and 100 uL DMEM and incubated for $10 \mathrm{~min}$ at room temperature before being added dropwise on the cells. Cells and viral supernatant were collected at $24 \mathrm{~h}$ post-transfection.

626 Cells were washed with $2 \mathrm{~mL}$ PBS and RNA was extracted with $1000 \mathrm{uL}$ TRI-reagent (Sigma627 Aldrich) according to the manufacturer's instructions. Viral supernatant was first clarified for 2 min 628 at $17000 \times g$, followed by a filtration step through 0.45 micron filter. The filtrate was then transferred into a new tube and the virus was pelleted for $2 \mathrm{~h}$ at $17000 \mathrm{x}$. The viral pellet was extracted with 
$630500 \mathrm{uL}$ TRI-reagent (Sigma-Aldrich) and DNA contaminants were removed by TurboDNase 631 (Invitrogen) treatment for 30 min at $37^{\circ} \mathrm{C}$. RNA was purified using NucleoSpin Gel and PCR Clean632 up kit (Macherey-Nagel) with NTC buffer (Macherey-Nagel) and eluted in $20 \mathrm{uL}$ of $5 \mathrm{mM}$ Tris- $\mathrm{HCl}$ $633 \mathrm{pH}$ 8.0. $10 \mathrm{uL}$ purified RNA was heat denatured at $65^{\circ} \mathrm{C}$ for 5 min together with $0.67 \mu \mathrm{M}$ reverse 634 primer (GATGGTTGTAGCTGTCCCAGTATTTGCC) and $1.67 \mathrm{mM}$ dNTPs in $15 \mathrm{uL}$ total volume, 635 then chilled on ice for 2 min. RNA was then reverse transcribed by adding 1X SSIV buffer, 5 mM 636 DTT, 20U RNasin, $100 \mathrm{U}$ SSIV in $25 \mu$ total volume and incubating at $52^{\circ} \mathrm{C}$ for $1 \mathrm{~h}$. cDNAs were

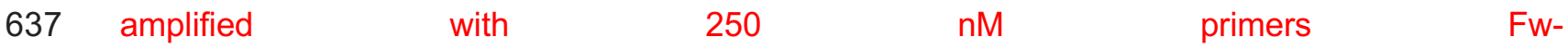
638 TCGTCGGCAGCGTCAGATGTGTATAAGAGACAGggtctctctggttagacc, Rv639 GTCTCGTGGGCTCGgAGATGTGTATAAGAGACAGGCGTACTCACCAGTCGCC, $\quad 200 \mu \mathrm{M}$ 640 dNTPs, 1X Q5 reaction buffer, and 0.02 U/uL of Q5 polymerase (NEB) using the PCR cycling 641 conditions: $98{ }^{\circ} \mathrm{C}$ for $1 \mathrm{~min}$, followed by 22 cycles of $98{ }^{\circ} \mathrm{C}$ for $10 \mathrm{~s}, 55^{\circ} \mathrm{C}$ for $20 \mathrm{~s}$, and $72{ }^{\circ} \mathrm{C}$ for $64230 \mathrm{~s}$. The PCR products were visualized by electrophoresis on $1 \%$ agarose gels in $1 \mathrm{X}$ TAE buffer 643 and column purified (using the NucleoSpin Gel and PCR Clean-up kit, Macherey-Nagel). 40 ng 644 purified products were used in the final indexing PCR using $2.5 \mathrm{uL}$ of Nextera DNA CD Indexes (96 645 Indexes, 96 Samples, Illumina) in a 14 uL reaction (200 $\mu \mathrm{M}$ dNTPs, 1X Q5 reaction buffer, and $6460.02 \mathrm{U} / \mathrm{uL}$ of Q5 polymerase (NEB)). The PCR cycling conditions were $98^{\circ} \mathrm{C}$ for $2 \mathrm{~min}$, followed by 6475 cycles of $98{ }^{\circ} \mathrm{C}$ for $30 \mathrm{~s}, 6255^{\circ} \mathrm{C}$ for $320 \mathrm{~s}$, and $72{ }^{\circ} \mathrm{C}$ for 1 min. Paired end PE150 sequencing 648 was carried out on an Miniseq instrument (Illumina) according to the manufacturer's instructions.

\section{Data availability}

650 The datasets generated during this study are available at the NCBI bioproject id PRJNA771368.

\section{Code availability}

652 Data were analyzed using publicly available tools as outlined in the material and methods.

\section{Acknowledgements}


654 We would like to thank Jean-Christophe Paillart and Roland Marquet for critical feedback. We also

655 thank Helmholtz Association (VH-NG-1347 to RS) and the Bundesministerium für Bildung und

656 Forschung (BMBF) (COMPLS-182 to RS and MvK). ASG was supported with a fellowship from the

657 Peter und Traudl Engelhorn Stiftung.

\section{Author contributions}

RPS, ASG and LY conceived the study. LY, ASG, CB, UBA, SA, and MON performed the

experiments. RPS, PB and MS performed the analysis. AK and NC purified Pr55 $5^{\mathrm{Gag}}$ and performed

MST measurements. RPS and LY wrote the manuscript with contributions from the other authors.

\section{Competing interests}

The authors declare they have no competing interests.

\section{Bibliography}

1. Paillart, J.-C., Shehu-Xhilaga, M., Marquet, R. \& Mak, J. Dimerization of retroviral RNA genomes: an inseparable pair. Nat. Rev. Microbiol. 2, 461-72 (2004).

2. Dubois, N., Marquet, R., Paillart, J.-C. \& Bernacchi, S. Retroviral RNA Dimerization: From Structure to Functions. Front. Microbiol. 9, 527 (2018).

3. Nikolaitchik, O. a et al. Probing the HIV-1 Genomic RNA Trafficking Pathway and Dimerization by Genetic Recombination and Single Virion Analyses. PLoS Pathog. 5, e1000627 (2009).

4. Sakuragi, J., Ueda, S., Iwamoto, A. \& Shioda, T. Possible role of dimerization in human immunodeficiency virus type 1 genome RNA packaging. J. Virol. 77, 4060-9 (2003).

5. Ooms, M., Huthoff, H., Russell, R., Liang, C. \& Berkhout, B. A riboswitch regulates RNA dimerization and packaging in human immunodeficiency virus type 1 virions. J. Virol. 78, 10814-9 (2004).

6. Russell, R. S. et al. Sequences downstream of the 5' splice donor site are required for both packaging and dimerization of human immunodeficiency virus type 1 RNA. J. Virol. 77, 84-96 (2003).

7. Nikolaitchik, O. A., Rhodes, T. D., Ott, D. \& Hu, W.-S. Effects of mutations in the human immunodeficiency virus type $1 \mathrm{Gag}$ gene on RNA packaging and recombination. J. Virol. 80, 46917 (2006). 
683

684

685

686

687

688

689

690

691

692

693

694

695

696

697

698

699

700

701

702

703

704

705

706

707

708

709

710

711

8. Moore, M. D. et al. Dimer initiation signal of human immunodeficiency virus type 1: its role in partner selection during RNA copackaging and its effects on recombination. J. Virol. 81, 400211 (2007).

9. Chen, J. et al. High efficiency of HIV-1 genomic RNA packaging and heterozygote formation revealed by single virion analysis. Proc. Natl. Acad. Sci. U. S. A. 106, 13535-40 (2009).

10. Smyth, R. P., Davenport, M. P. \& Mak, J. The origin of genetic diversity in HIV-1. Virus Res. 169, 415-29 (2012).

11. Lu, K. et al. NMR detection of structures in the HIV-1 5'-leader RNA that regulate genome packaging. Science 334, 242-5 (2011).

12. Keane, S. C. et al. RNA structure. Structure of the HIV-1 RNA packaging signal. Science 348, 917-21 (2015).

13. Kharytonchyk, S. et al. Transcriptional start site heterogeneity modulates the structure and function of the HIV-1 genome. Proc. Natl. Acad. Sci. U. S. A. 201616627 (2016) doi:10.1073/pnas.1616627113.

14. Brown, J. D. et al. Structural basis for transcriptional start site control of HIV-1 RNA fate. Science 368, 413-417 (2020).

15. Huthoff, H. \& Berkhout, B. Two alternating structures of the HIV-1 leader RNA. RNA 7, 14357 (2001).

16. Abbink, T. E. M., Ooms, M., Haasnoot, P. C. J. \& Berkhout, B. The HIV-1 Leader RNA Conformational Switch Regulates RNA Dimerization but Does Not Regulate mRNA Translation †. Biochemistry 44, 9058-9066 (2005).

17. Skripkin, E. et al. Identification of the primary site of the human immunodeficiency virus type 1 RNA dimerization in vitro. Proc. Natl. Acad. Sci. U. S. A. 91, 4945-9 (1994).

18. Paillart, J. C., Marquet, R., Skripkin, E., Ehresmann, B. \& Ehresmann, C. Mutational analysis of the bipartite dimer linkage structure of human immunodeficiency virus type 1 genomic RNA. J. Biol. Chem. 269, 27486-93 (1994).

19. Paillart, J. C. et al. A dual role of the putative RNA dimerization initiation site of human immunodeficiency virus type 1 in genomic RNA packaging and proviral DNA synthesis. J. Virol. 70, 8348-54 (1996).

20. Muriaux, D., De Rocquigny, H., Roques, B. P. \& Paoletti, J. NCp7 activates HIV-1Lai RNA dimerization by converting a transient loop-loop complex into a stable dimer. J. Biol. Chem. 271, 33686-92 (1996).

21. Fu, W., Gorelick, R. J. \& Rein, A. Characterization of human immunodeficiency virus type 1 dimeric RNA from wild-type and protease-defective virions. J. Virol. 68, 5013-5018 (1994).

22. Jalalirad, M. \& Laughrea, M. Formation of immature and mature genomic RNA dimers in wild-type and protease-inactive HIV-1: differential roles of the Gag polyprotein, nucleocapsid proteins NCp15, NCp9, NCp7, and the dimerization initiation site. Virology 407, 225-36 (2010).

23. Russell, R. S., Hu, J., Laughrea, M., Wainberg, M. a. \& Liang, C. Deficient dimerization of human immunodeficiency virus type 1 RNA caused by mutations of the u5 RNA sequences. Virology 303, 152-63 (2002). 
24. Shen, N., Jetté, L., Wainberg, M. A. \& Laughrea, M. Role of stem B, loop B, and nucleotides next to the primer binding site and the kissing-loop domain in human immunodeficiency virus type 1 replication and genomic-RNA dimerization. J. Virol. 75, 10543-9 (2001).

25. Marquet, R., Paillart, J. C., Skripkin, E., Ehresmann, C. \& Ehresmann, B. Dimerization of human immunodeficiency virus type 1 RNA involves sequences located upstream of the splice donor site. Nucleic Acids Res. 22, 145-51 (1994).

26. Song, R., Kafaie, J. \& Laughrea, M. Role of the 5' TAR stem--loop and the U5-AUG duplex in dimerization of HIV-1 genomic RNA. Biochemistry 47, 3283-93 (2008).

27. Abbink, T. E. M. \& Berkhout, B. A novel long distance base-pairing interaction in human immunodeficiency virus type 1 RNA occludes the Gag start codon. J. Biol. Chem. 278, 11601-11 (2003).

28. Kenyon, J. C., Prestwood, L. J., Le Grice, S. F. J. \& Lever, A. M. L. In-gel probing of individual RNA conformers within a mixed population reveals a dimerization structural switch in the HIV-1 leader. Nucleic Acids Res. 41, e174 (2013).

29. Obayashi, C. M., Shinohara, Y., Masuda, T. \& Kawai, G. Influence of the 5'-terminal sequences on the 5'-UTR structure of HIV-1 genomic RNA. Sci. Rep. 11, 1-9 (2021).

30. Lu, K., Heng, X. \& Summers, M. F. Structural determinants and mechanism of HIV-1 genome packaging. J. Mol. Biol. 410, 609-633 (2011).

31. Brigham, B. S., Kitzrow, J. P., Reyes, J.-P. C., Musier-Forsyth, K. \& Munro, J. B. Intrinsic conformational dynamics of the HIV-1 genomic RNA 5'UTR. Proc. Natl. Acad. Sci. 116, 10372 10381 (2019).

32. Abd El-Wahab, E. W. et al. Specific recognition of the HIV-1 genomic RNA by the Gag precursor. Nat. Commun. 5, 4304 (2014).

33. Mailler, E. et al. The Life-Cycle of the HIV-1 Gag-RNA Complex. Viruses 8, 248 (2016).

34. Freed, E. O. HIV-1 assembly, release and maturation. Nat. Rev. Microbiol. 13, 484-96 (2015).

35. Sakuragi, J. I. \& Panganiban, a T. Human immunodeficiency virus type 1 RNA outside the primary encapsidation and dimer linkage region affects RNA dimer stability in vivo. J. Virol. 71, 3250-4 (1997).

36. Jones, K. L., Sonza, S. \& Mak, J. Primary T-lymphocytes rescue the replication of HIV-1 DIS RNA mutants in part by facilitating reverse transcription. Nucleic Acids Res. 36, 1578-88 (2008).

37. Clever, J. L. \& Parslow, T. G. Mutant human immunodeficiency virus type 1 genomes with defects in RNA dimerization or encapsidation. J. Virol. 71, 3407-14 (1997).

38. Smyth, R. P. et al. Mutational interference mapping experiment (MIME) for studying RNA structure and function. Nat. Methods 12, 866-872 (2015).

39. Smyth, R. P. et al. In cell mutational interference mapping experiment (in cell MIME) identifies the 5' polyadenylation signal as a dual regulator of HIV-1 genomic RNA production and packaging. Nucleic Acids Res. 46, e57-e57 (2018). 
40. Pop, C. et al. Causal signals between codon bias, mRNA structure, and the efficiency of translation and elongation. Mol. Syst. Biol. 10, 770 (2014).

764

765

766

767

768

769

770

771

772

773

774

775

776

777

778

779

780

781

782

783

784

785

786

787

788

789

790

791

792

793

794

795

796

797

798

799

41. Cordero, P., Kladwang, W., Vanlang, C. C. \& Das, R. Quantitative dimethyl sulfate mapping for automated RNA secondary structure inference. Biochemistry 51, 7037-7039 (2012).

42. Mailler, E., Paillart, J.-C., Marquet, R., Smyth, R. P. \& Vivet-Boudou, V. The evolution of RNA structural probing methods: From gels to next-generation sequencing. Wiley Interdiscip. Rev. RNA 10, (2019).

43. Rouskin, S., Zubradt, M., Washietl, S., Kellis, M. \& Weissman, J. S. Genome-wide probing of RNA structure reveals active unfolding of mRNA structures in vivo. Nature 505, 701-5 (2014).

44. Zubradt, M. et al. DMS-MaPseq for genome-wide or targeted RNA structure probing in vivo. Nat. Methods 14, 75-82 (2017).

45. Tian, S. \& Das, R. RNA structure through multidimensional chemical mapping. Q. Rev. Biophys. 49, e7 (2016).

46. Cheng, C. Y., Kladwang, W., Yesselman, J. D. \& Das, R. RNA structure inference through chemical mapping after accidental or intentional mutations. Proc. Natl. Acad. Sci. U. S. A. 114, 9876-9881 (2017).

47. Gavazzi, C. et al. A functional sequence-specific interaction between influenza A virus genomic RNA segments. Proc. Natl. Acad. Sci. U. S. A. 110, 16604-9 (2013).

48. Gavazzi, C. et al. An in vitro network of intermolecular interactions between viral RNA segments of an avian H5N2 influenza A virus: Comparison with a human H3N2 virus. Nucleic Acids Res. 41, 1241-1254 (2013).

49. Fajardo, T., Sung, P.-Y., Celma, C. C. \& Roy, P. Rotavirus Genomic RNA Complex Forms via Specific RNA-RNA Interactions: Disruption of RNA Complex Inhibits Virus Infectivity. Viruses 9, (2017).

50. Masuda, T. et al. Fate of HIV-1 cDNA intermediates during reverse transcription is dictated by transcription initiation site of virus genomic RNA. Sci. Rep. 5, 1-15 (2015).

51. Busan, S. \& Weeks, K. M. Accurate detection of chemical modifications in RNA by mutational profiling (MaP) with ShapeMapper 2. RNA (2017) doi:10.1261/rna.061945.117.

52. Goldschmidt, V. et al. Structural variability of the initiation complex of HIV-1 reverse transcription. J. Biol. Chem. 279, 35923-35931 (2004).

53. Goldschmidt, V. et al. Direct and indirect contributions of RNA secondary structure elements to the initiation of HIV-1 reverse transcription. J. Biol. Chem. 277, 43233-43242 (2002).

54. Incarnato, D., Morandi, E., Simon, L. M. \& Oliviero, S. RNA Framework: an all-in-one toolkit for the analysis of RNA structures and post-transcriptional modifications. Nucleic Acids Res. 46, e97 (2018).

55. Lorenz, R. et al. ViennaRNA Package 2.0. Algorithms Mol. Biol. 6, 26 (2011).

56. Nikolaitchik, O. A. et al. Unpaired Guanosines in the 5' Untranslated Region of HIV-1 RNA Act Synergistically To Mediate Genome Packaging. J. Virol. 94, (2020). 
57. Deigan, K. E., Li, T. W., Mathews, D. H. \& Weeks, K. M. Accurate SHAPE-directed RNA structure determination. Proc. Natl. Acad. Sci. U. S. A. 106, 97-102 (2009).

58. Kladwang, W., VanLang, C. C., Cordero, P. \& Das, R. A two-dimensional mutate-and-map strategy for non-coding RNA structure. Nat. Chem. 3, 954-62 (2011).

59. Clever, J. L., Wong, M. L. \& Parslow, T. G. Requirements for kissing-loop-mediated dimerization of human immunodeficiency virus RNA. J. Virol. 70, 5902-5908 (1996).

60. Huthoff, H., Bugala, K., Barciszewski, J. \& Berkhout, B. On the importance of the primer activation signal for initiation of tRNA(lys3)-primed reverse transcription of the HIV-1 RNA genome. Nucleic Acids Res. 31, 5186-94 (2003).

61. Ooms, M., Verhoef, K., Southern, E., Huthoff, H. \& Berkhout, B. Probing alternative foldings of the HIV-1 leader RNA by antisense oligonucleotide scanning arrays. Nucleic Acids Res. 32, 81927 (2004).

62. Kasprzak, W., Bindewald, E. \& Shapiro, B. a. Structural polymorphism of the HIV-1 leader region explored by computational methods. Nucleic Acids Res. 33, 7151-63 (2005).

63. Wilkinson, K. a et al. High-throughput SHAPE analysis reveals structures in HIV-1 genomic RNA strongly conserved across distinct biological states. PLoS Biol. 6, e96 (2008).

64. Watts, J. M. et al. Architecture and secondary structure of an entire HIV-1 RNA genome. Nature 460, 711-6 (2009).

65. Siegfried, N. A., Busan, S., Rice, G. M., Nelson, J. A. E. \& Weeks, K. M. RNA motif discovery by SHAPE and mutational profiling (SHAPE-MaP). Nat. Methods (2014) doi:10.1038/nmeth.3029.

66. Paillart, J. C. et al. First snapshots of the HIV-1 RNA structure in infected cells and in virions. J. Biol. Chem. 279, 48397-48403 (2004).

67. Balvay, L., Lastra, M. L., Sargueil, B., Darlix, J. L. \& Ohlmann, T. Translational control of retroviruses. Nat. Rev. Microbiol. 5, 128-140 (2007).

68. Bernacchi, S. et al. HIV-1 Pr55 Gag binds genomic and spliced RNAs with different affinity and stoichiometry. RNA Biol. 14, 90-103 (2017).

69. Houzet, L. et al. HIV controls the selective packaging of genomic, spliced viral and cellular RNAs into virions through different mechanisms. Nucleic Acids Res. 35, 2695-704 (2007).

70. Seif, E., Niu, M. \& Kleiman, L. Annealing to sequences within the primer binding site loop promotes an HIV-1 RNA conformation favoring RNA dimerization and packaging. RNA 19, 138493 (2013).

71. Gibbs, J. S., Regier, D. A. \& Desrosiers, R. C. Construction and In Vitro Properties of HIV1 Mutants with Deletions in 'Nonessential' Genes. AIDS Res. Hum. Retroviruses 10, 343-350 (1994).

72. McKinstry, W. J. et al. Expression and purification of soluble recombinant full length HIV-1 Pr55(Gag) protein in Escherichia coli. Protein Expr. Purif. 100, 10-8 (2014).

73. Smith, M. R., Smyth, R. P., Marquet, R. \& von Kleist, M. MIMEAnTo: profiling functional RNA in mutational interference mapping experiments. Bioinformatics 32, 3369-3370 (2016). 
839 74. Incarnato, D., Neri, F., Anselmi, F. \& Oliviero, S. RNA structure framework: automated 840 transcriptome-wide reconstruction of RNA secondary structures from high-throughput structure 841 probing data. Bioinformatics 32, 459-61 (2016).

842 75. Smola, M. J., Calabrese, J. M. \& Weeks, K. M. Detection of RNA-Protein Interactions in 843 Living Cells with SHAPE. Biochemistry 54, 6867-6875 (2015).

844 76. Darty, K., Denise, A. \& Ponty, Y. VARNA: Interactive drawing and editing of the RNA 845 secondary structure. Bioinformatics 25, 1974-5 (2009).

$84677 . \quad$ Cordero, P., Lucks, J. B. \& Das, R. An RNA Mapping DataBase for curating RNA structure 847 mapping experiments. Bioinformatics 28, 3006-8 (2012). 
a

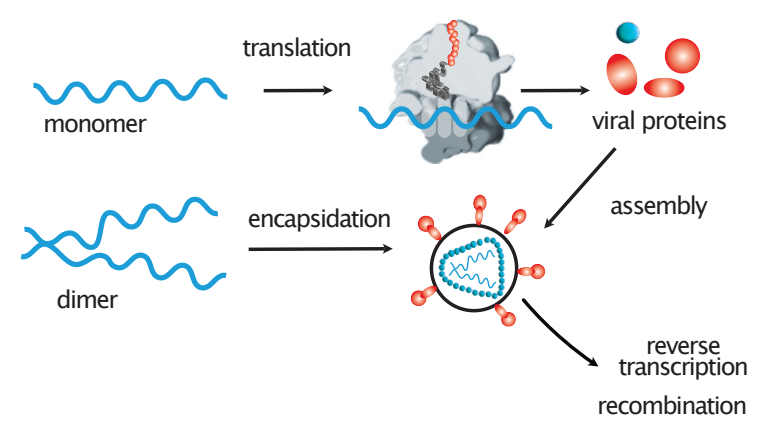

C
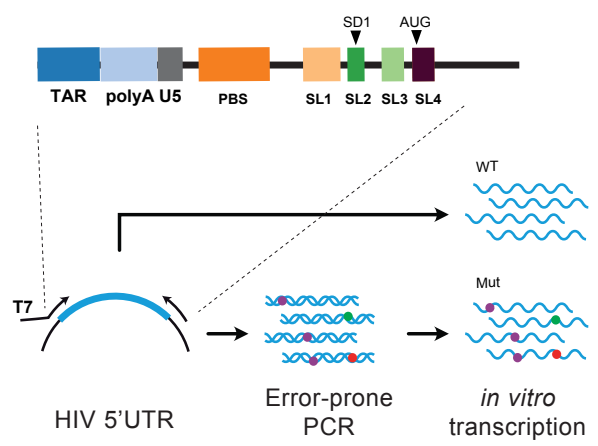

HIV 5'UTR b

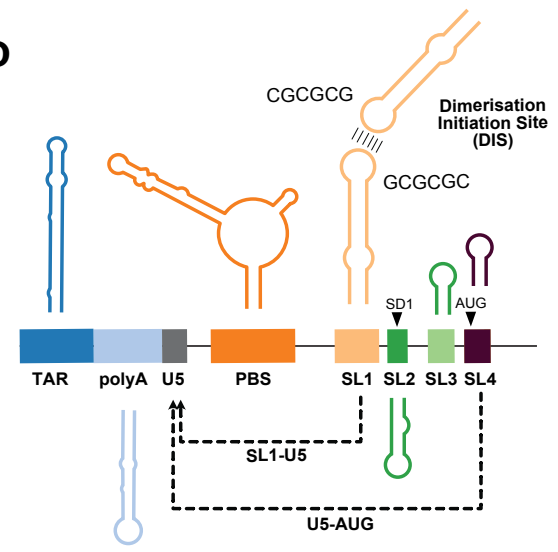

d

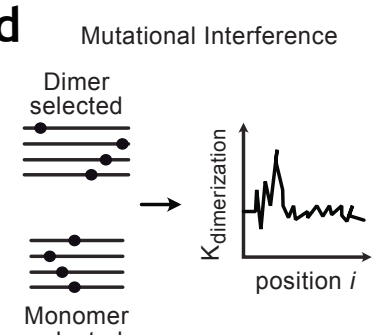

e

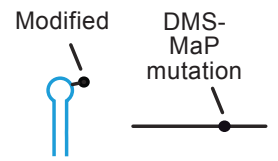

salt

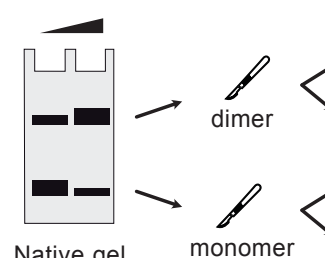

Native gel

electrophoresis

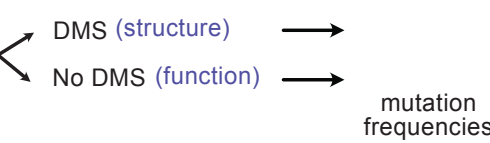

DMS (structure)<smiles>C1CCCCC1</smiles>
requencies f $\begin{array}{r}\text { Two-dimensional } \\ \text { structural probing }\end{array}$

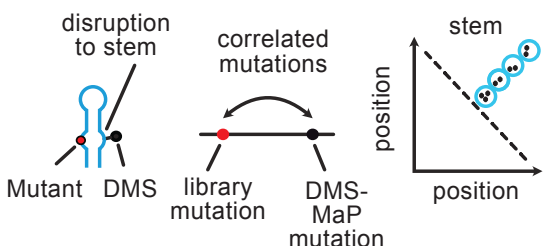




\section{Figure 1}

2 HIV-1 dimerization and its analysis (a) Dimerization is a key step in the HIV-1 life cycle. Monomeric

3 RNA is thought to be preferentially translated, in contrast to dimeric RNA which is a pre-requisite

4 for packaging into virions. Dimeric RNA helps maintain genome integrity through recombination (b)

5 The HIV-15'UTR is composed of distinct structural domains linked to different functions in the HIV-

61 life cycle. TAR for transcription. PolyA for polyadenylation which is inactive in the 5'UTR. U5 in

7 unique 5 region, PBS, or primer binding site, for annealing of the host tRNA for initiating reverse

8 transcription. SL1-SL3 contain the packaging signal. SL2 contains the splice donor site.

9 Dimerization occurs through a kissing loop interaction at a sequence in SL1. Long distance interactions / alternative folds involving long distance interactions, such as between SL1-U5 and U5-AUG may regulate dimerization. (c) Functional Analysis of RNA Structure (FARS-seq). Mutant RNA sequences are generated by mutagenic PCR and in vitro transcription. Mutant populations are physically separated into monomer and dimer fractions and probed with DMS or left untreated. Mutation frequencies are analyzed by next generation sequencing. (d) Functional profiles are obtained by mutational interference. $\mathrm{K}^{\text {dimer }}$ is a quantitative measure of dimerization based on the ratio of mutations in the dimer selected vs monomer selected population, corrected for mutations introduced during the library preparation and sequencing (e) Structural profiles are obtained by DMS which specifically reacts with unpaired $A$ and $C$ residues. DMS-MaP-seq measures DMS reactivities as mutation rates in DMS treated vs untreated controls (2) Two-dimensional analysis identifies RNA stems through correlations between stem-disrupting mutations and mutations 21 induced by DMS. 
a

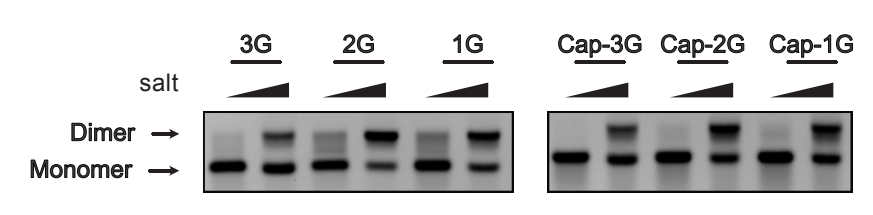

C

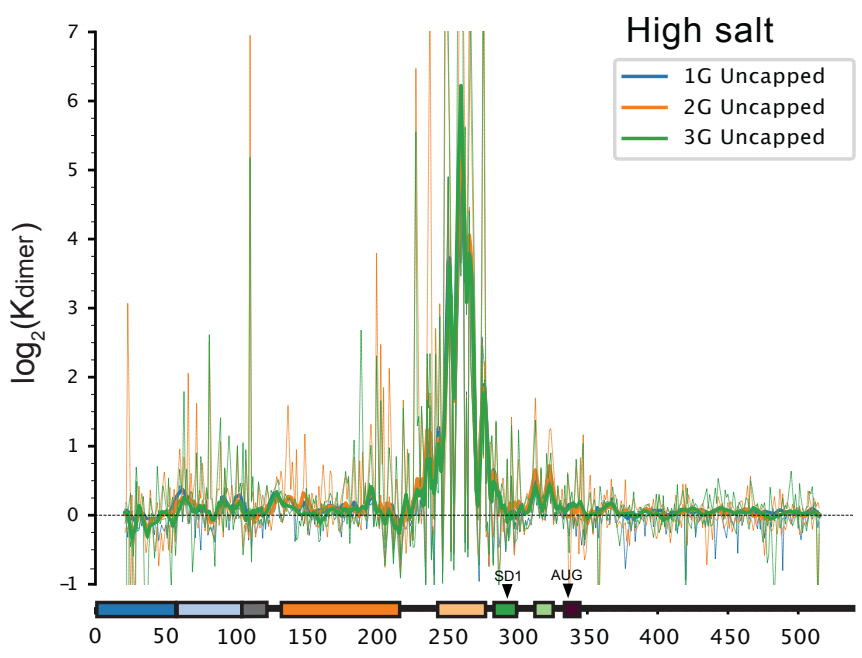

b

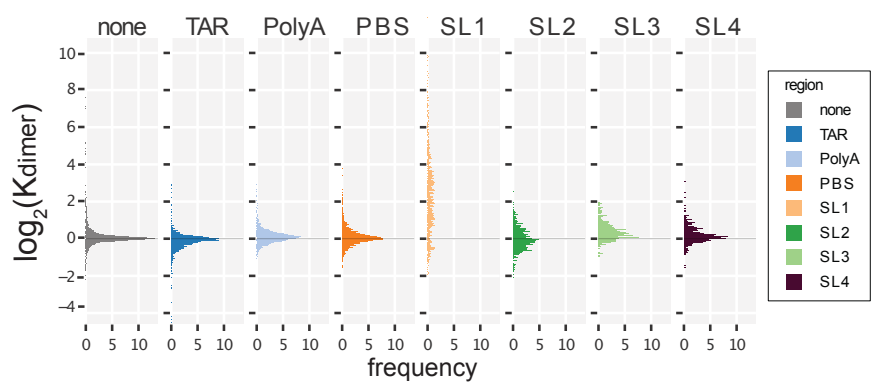

d

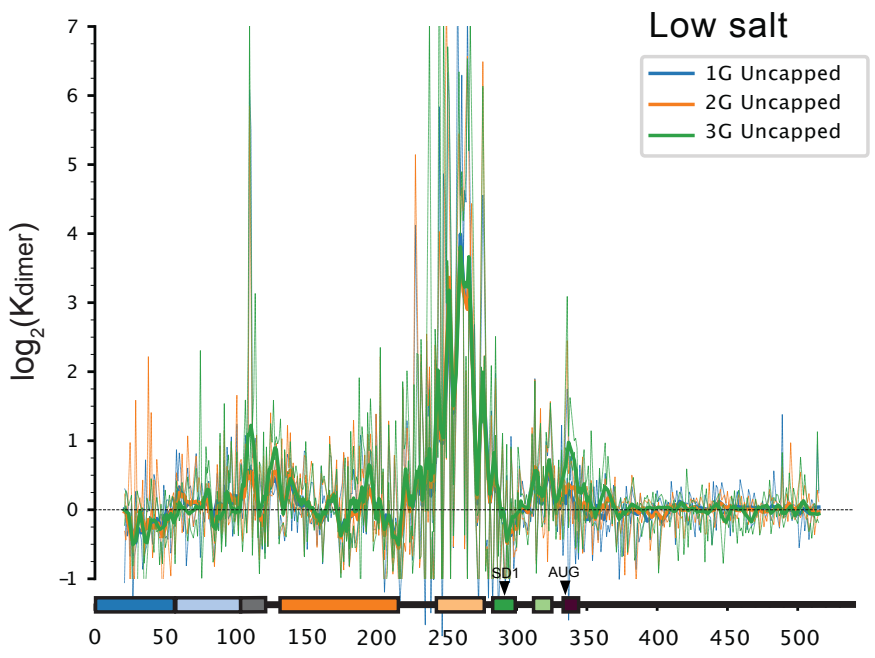

High salt

e

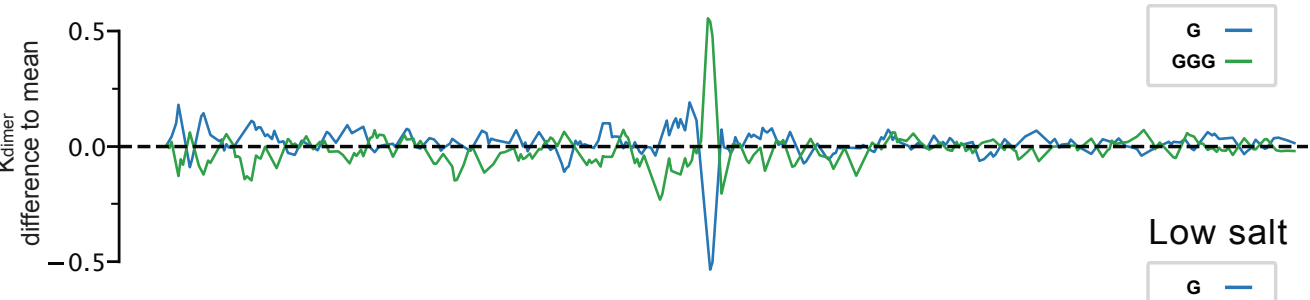

f

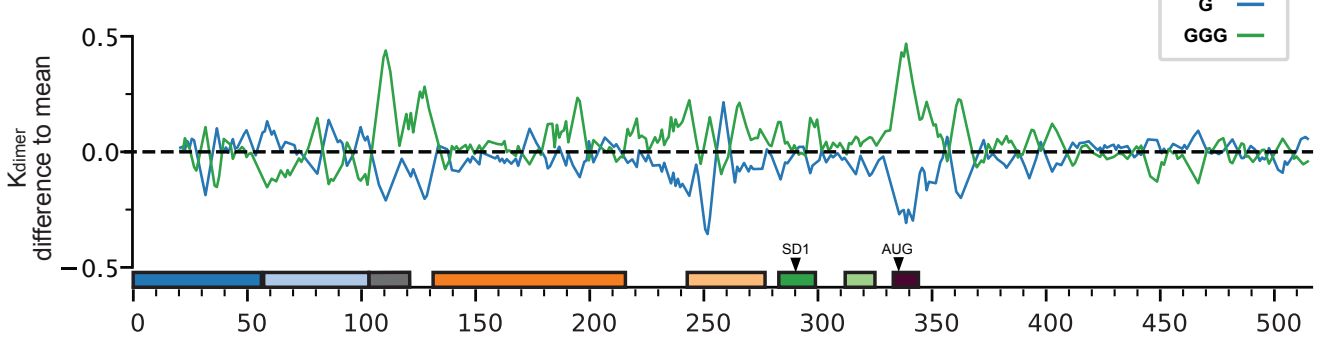




\section{Figure 2}

23 Functional profiling of sequences involved in dimerization by mutational interference (a) 1G, 2G, 24 and $3 G$ capped and uncapped transcript variants migrate as distinct monomer and dimer bands on native agarose gels in both low salt and high salt buffers $(\mathbf{b}-\mathbf{h}) \mathrm{k}^{\text {dimer }}$ is a relative measure of the effects of a mutation on dimerization, calculated as the ratio of mutation frequencies in the monomer vs the dimerized RNA, and corrected for errors introduced during library preparation and sequencing. (b) $\log _{2}\left(\mathrm{k}^{\text {dimer }}\right)$ values binned according to functional domain in the 5'UTR: TAR, U5, PBS, SL1, SL2, SL3 and SL4. None refers to nucleotide positions that do not fall into any structural domain. (c-d) median $\log _{2}\left(\mathrm{k}^{\text {dimer }}\right)$ values for each genome position for all three uncapped transcript variants in high and low salt buffers. Lines represent median $\log _{2}\left(k^{\text {dimer }}\right)$ values smoothed with a window size of 5 nt. $(\mathbf{e}-\mathbf{h}) \log _{2}\left(\mathrm{k}^{\text {dimer }}\right)$ values of the $1 \mathrm{G}$ and $3 \mathrm{G}$ transcript variants compared to the mean of the 1G, $2 \mathrm{G}$ and $3 \mathrm{G}$ transcripts in high and low salt buffers. 
a
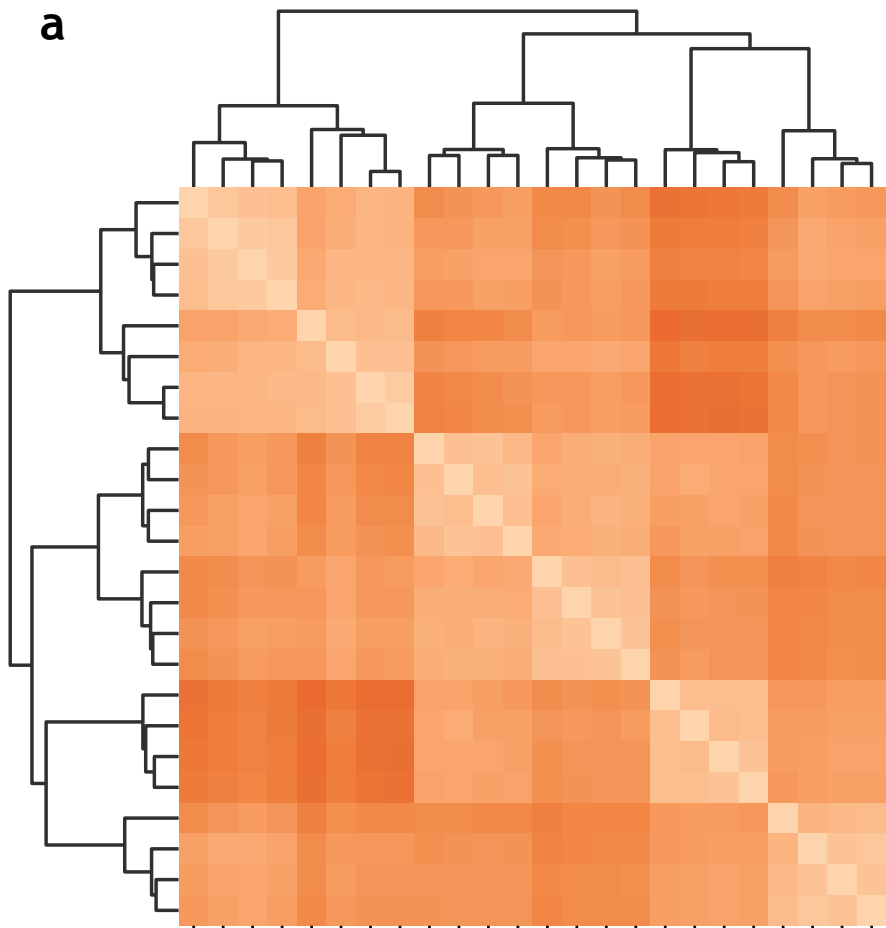

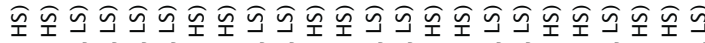

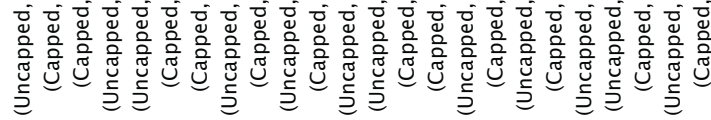

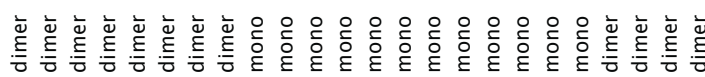

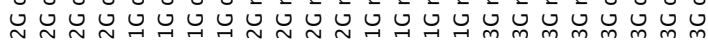

d

\section{z-factor}

absolute threshold

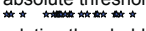

relative threshold

TAR PolyA U5

PBS

SL1 SL2 SL3 SL4

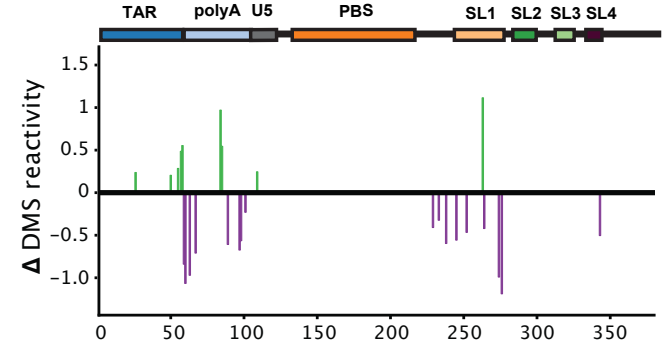

1G/2G dimer vs $3 \mathrm{G}$ monomer
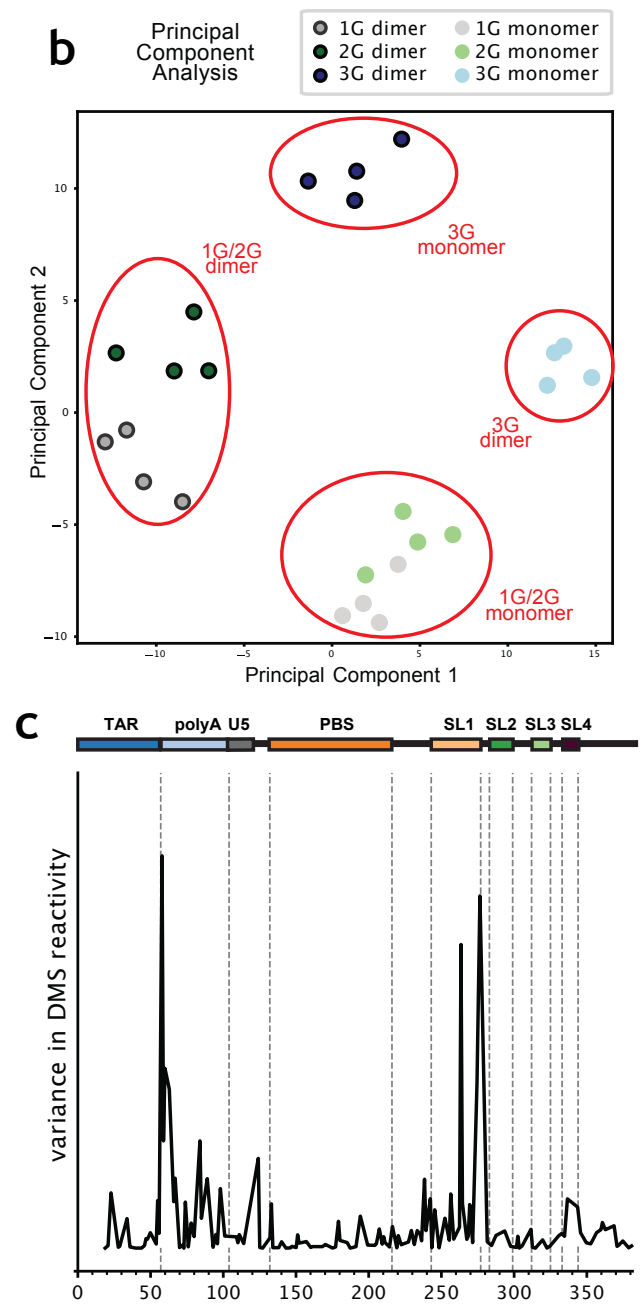

- 3G mono (Uncapped, LS)

- 3C mono (Capped, HS)

- 3G mono (Uncapped, HS)

- 3G dimer (Uncapped, LS)

- 3G dimer (Capped, HS)

- 3G dimer (Uncapped, HS)

3G dimer (Capped, LS) 


\section{$34 \quad$ Figure 3}

35 Structural profiling reveals identifies structural conformations of the HIV-1 5'UTR. (a) Clustering of Kendal rank correlations of DMS reactivities across all positions reveals structural relationships between monomer and dimer isolated populations from uncapped and capped transcript variants in high and low salt buffer. Relationships between sample DMS reactivities was determined by hierarchical clustering using the 'average' linking method (b) Principal component analysis of DMS reactivities identifies structural four structural classes of the HIV-1 5'UTR (c) Variance in DMS reactivities across genome positions from all samples is enriched at SL1 and TAR/polyA boundary (d) Statistical analysis DMS reactivities in 1G/2G and 3G structural classes find significant differences in reactivities are mainly localized to polyA and SL1. A z-factor test identifies nucleotides where DMS reactivities change by $>1.96$ standard deviations of the DMS errors. An absolute difference threshold ensures that a minimum reactivity change of 0.2 is needed for the site to be considered biologically relevant. The relative threshold of 0.75 -fold was used to remove

47 false positives where DMS reactivities are high in both conditions such that a large change in reactivity is unlikely to affect RNA structure. 

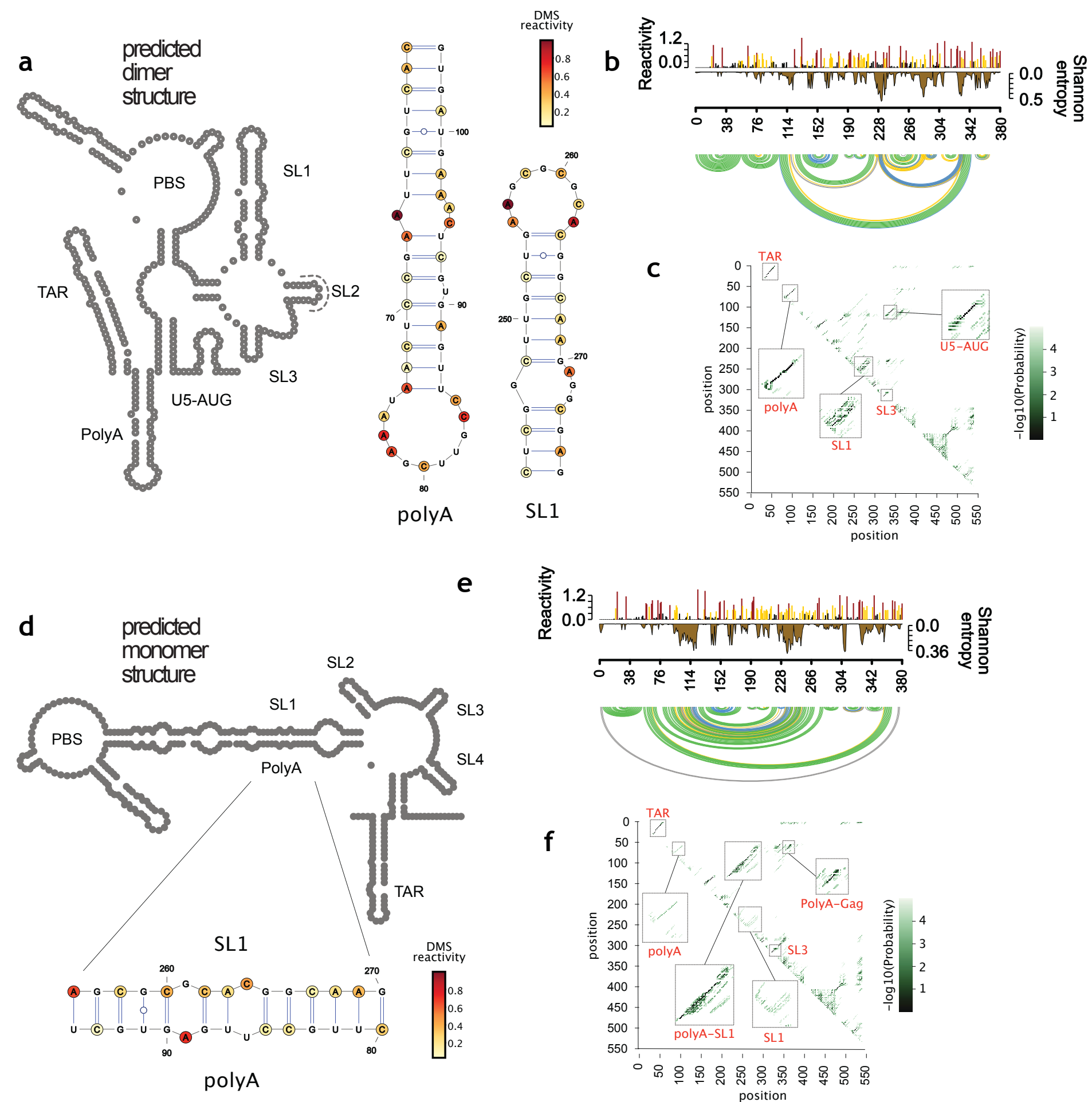


\section{$49 \quad$ Figure 4}

50 Secondary structure model for $1 \mathrm{G} / 2 \mathrm{G}$ dimer and $3 \mathrm{G}$ monomer populations. (a, d) Secondary 51 structure model of dimer and monomer class, respectively. Models were obtained using DMS reactivities as soft constraints for in silico folding in the Vienna RNA structure package. For the

53 dimer structure, the U1sRNA binding site within SL2 is shown. Structures of polyA and SL1 stem loops and polyA-SL1 interaction are shown. DMS reactivities from dimer samples were mapped to A and $C$ residues of the polyA and SL1 stem loop structures. DMS reactivites from the monomer 56 samples were mapped to $A$ and $C$ residues of the polyA-SL1 interaction. Red signifies highly reactive positions that are unpaired. Pale yellow signifies unreactive positions that are base-paired. (b, e) DMS reactivities and Shannon entropies for the 1G/2G dimer and $3 G$ monomer class, respectively. Arc plots show base pairing probabilities (green=70-100\%; blue=40-70\%; yellow=1040\%; grey=5-10\%). Grey bar in (e) signifies polyA-SL1 interaction. (c, d) Dot plots of RNA base pairing probabilities for the $1 \mathrm{G} / 2 \mathrm{G}$ dimer and $3 \mathrm{G}$ monomer class, respectively, reveal alternative folding possibilities. RNA stems are shown along the diagonals. 
a two-dimensional RNA structural probing
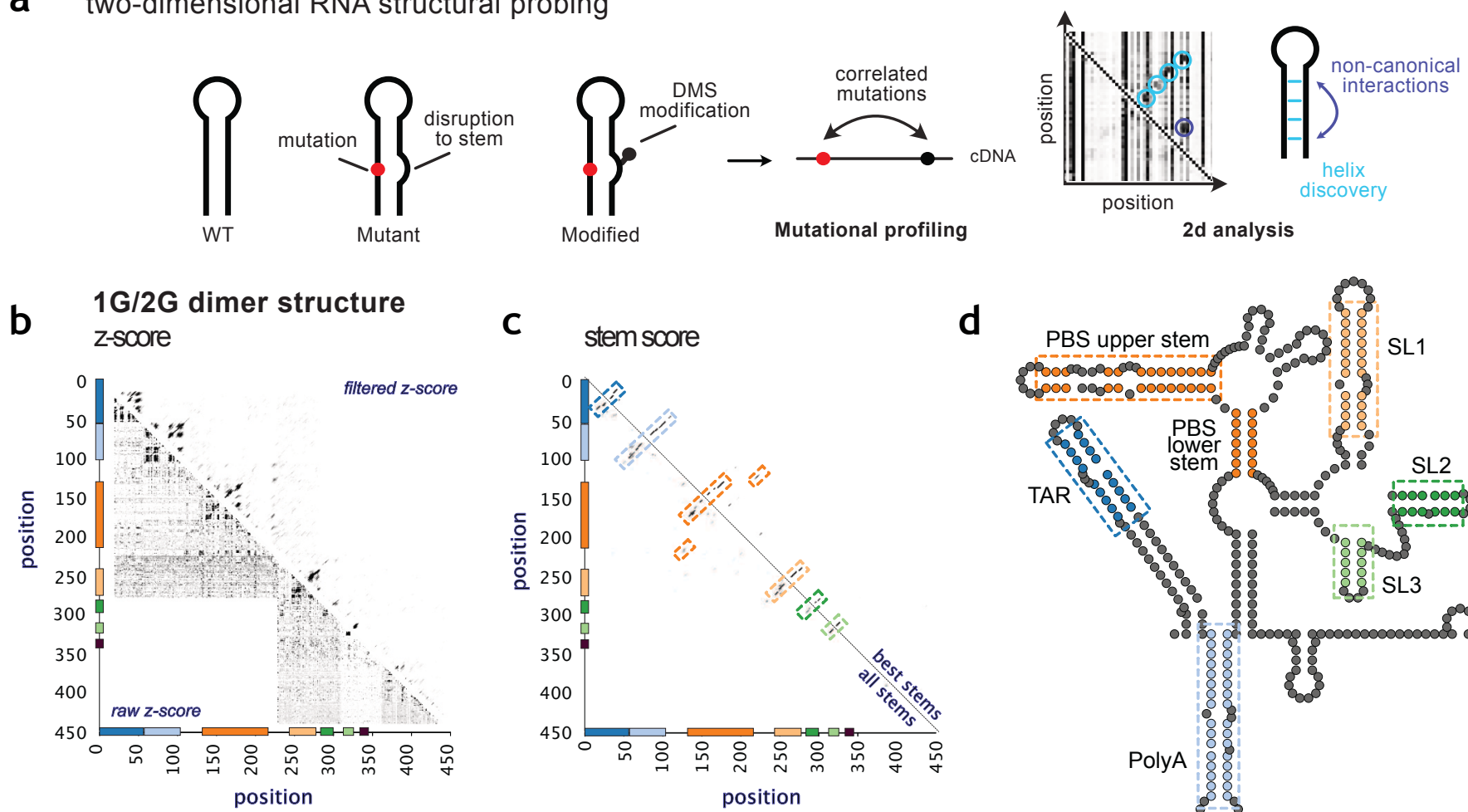

C
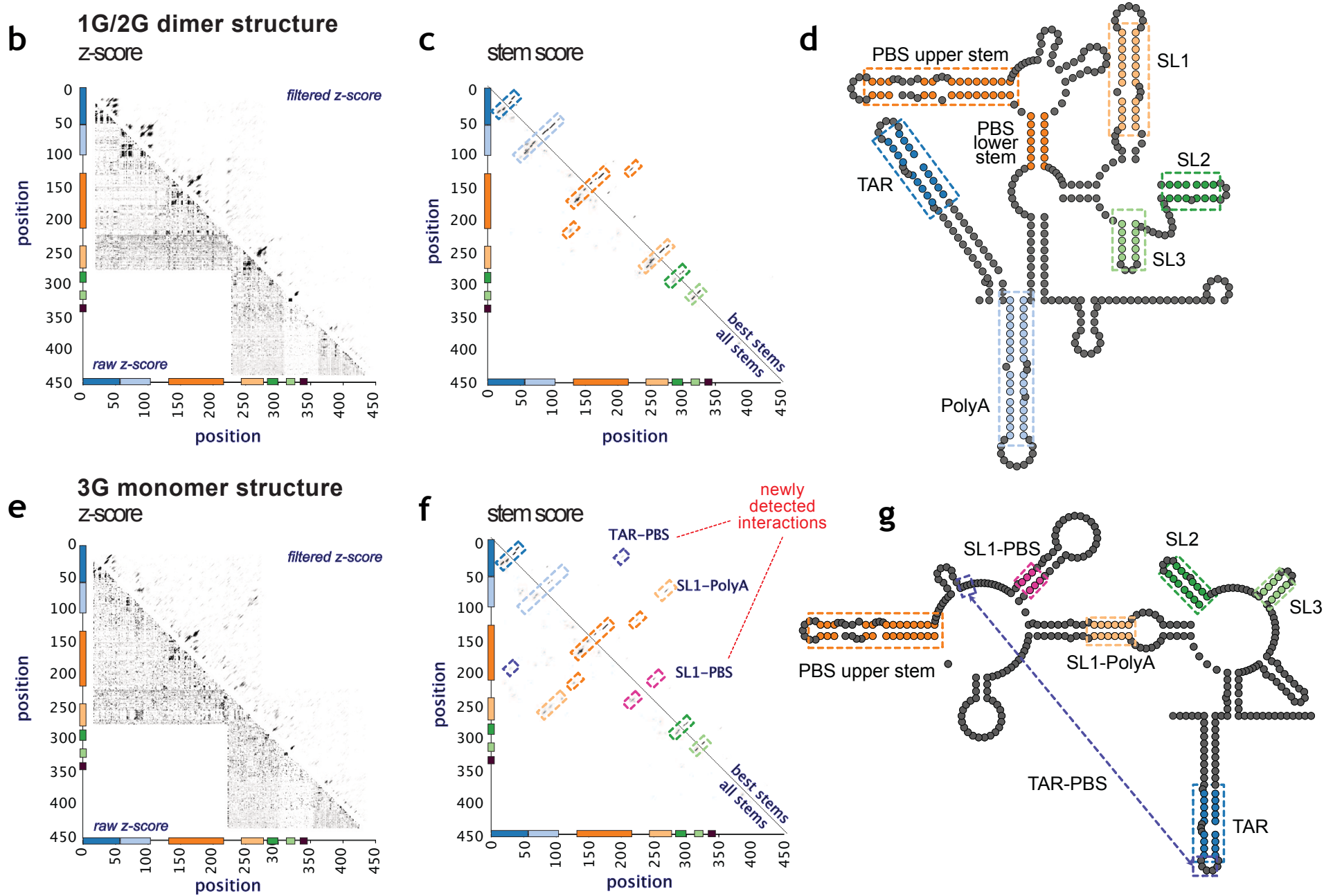
Two-dimensional mapping of RNA structure in 1G/2G dimer and 3G monomer populations. (a) Mutations disrupting RNA stems lead to increases in DMS reactivity at positions opposite the mutation. Positions of DMS modification are read out as mutations leading to correlated mutations at pairs of nucleotides involved in RNA structure. RNA secondary structures (blue circles) are identified along the diagonals. Punctate signals (purple circles) can signify non-canonical or tertiary interactions. (b, e) z-score analysis of mutation frequencies from $1 G / 2 G$ dimer and $3 G$ monomer populations, respectively. Raw Z-scores (lower diagonal) reveal pairs of positions enriched with mutations. Filtered z-scores (upper diagonal) enhance stem signals by applying a convolution filter and signal threshold. Insets are zooms of the filtered z-scores for the polyA-SL1, PBS-SL1 and SL1 stems (c, f) Stem detection in 1G/2G dimer and 3G monomer populations, respectively. All stems (lower diagonal) reveal all possible stems of minimum length 3 by applying a filter for WatsonCrick and Wobble base pairs to the filtered Z-score. Best stem (upper diagonal) selects the best non-conflicting stems by removing conflicting stems based on filtered Z-score. Coloured boxes represent regions that are highlighted in enhance RNA secondary structure models $(\mathbf{d}, \mathbf{g})$ Enhanced RNA secondary structure models of $1 \mathrm{G} / 2 \mathrm{G}$ dimer and $3 \mathrm{G}$ monomer populations, respectively. Coloured base pairings were detected in multi-dimensional mapping and used as hard constraints before in RNA secondary structure prediction. Dark blue is TAR. Light blue is polyA. Orange is PBS. Mustard is SL1. Dark green is SL2. Light green is SL3. Red represents the polyASL1 interactions, Pink the novel SL1-PBS interaction, and purple the TAR-PBS interaction. 

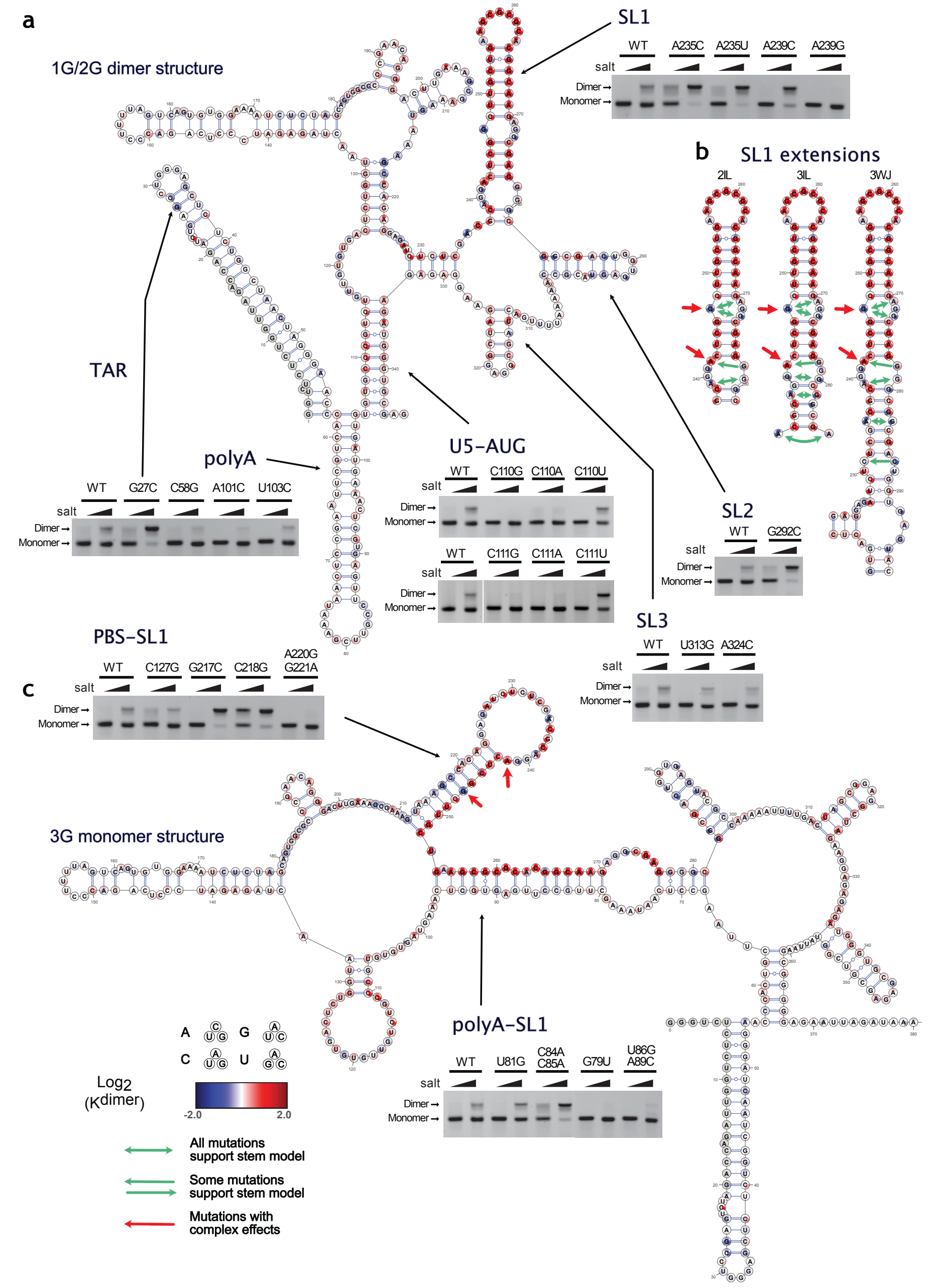


\section{$84 \quad$ Figure 6}

85 Structure/function analysis of HIV-1 dimerization. Single nucleotide resolution functional profiling data mapped the (a) dimer and (c) monomer structures expressed as $\log _{2}\left(K^{\text {dimer }}\right)$ values. Each individual mutant shown as one of three circle in the order $A, C, G, U$ clockwise from upper position

88 (excluding the WT base). Validation of structural models on 3G RNA by point mutagenesis followed by native agarose gel electrophoresis in two different buffer conditions. Red circles show mutations inhibiting dimerization, and blue circles show mutations enhancing dimerization. $\log _{2}\left(K^{\text {dimer }}\right)$ values above 2 are capped. (b) Functional profiling data mapped to different structural models of SL1 containing mutually exclusive internal loop configurations. Two internal loop (2IL), three internal loop (3IL), and the three-way junction (3WJ) are mutually exclusive models of SL1 structure based on chemical probing or biophysical measurements. Green arrows show mutations that improve dimerization by closing or reducing the size of internal loops, providing evidence that SL1 is metastable, and that alternative SL1 conformations can form and dimerize. Red arrows show

97 mutations that have complex effects on dimerization because they impact the novel PBS-SL1 98 interaction. 
a
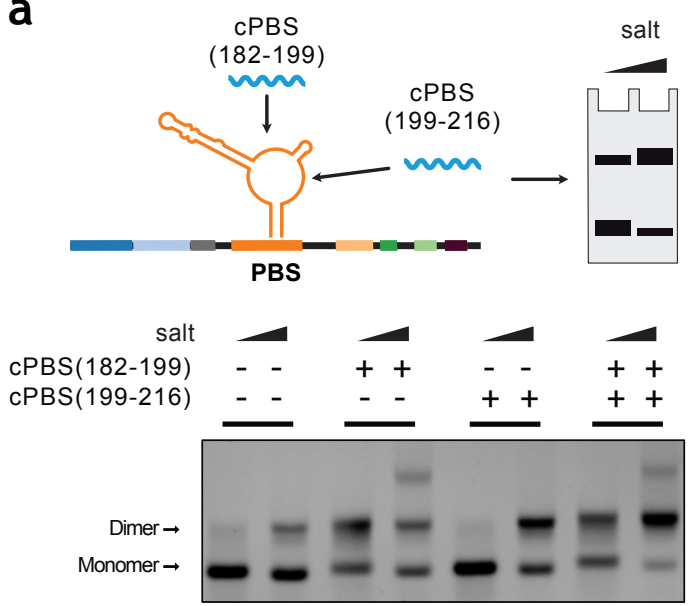

C

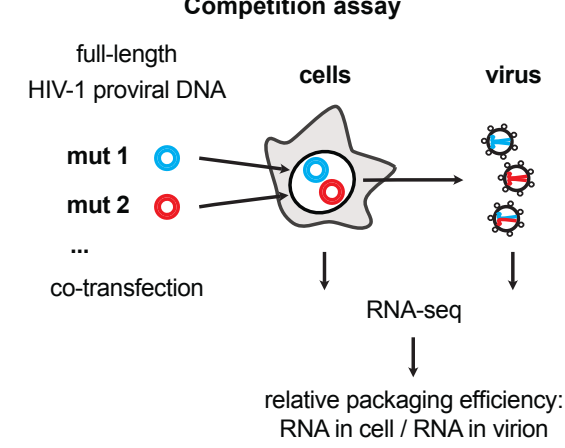

d

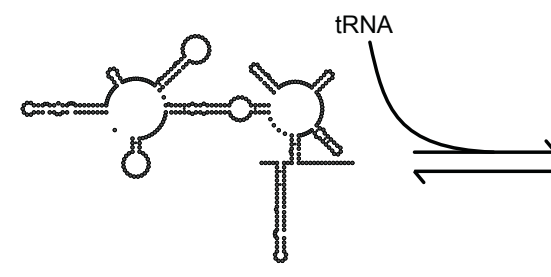

monomer b

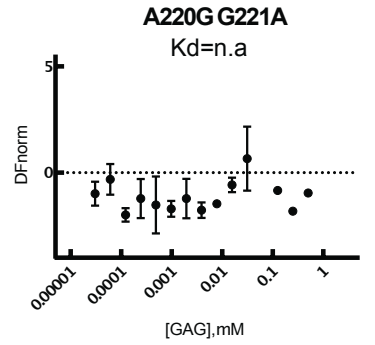

C218G

$\mathrm{Kd}=19 \pm 16 \mathrm{nM}$
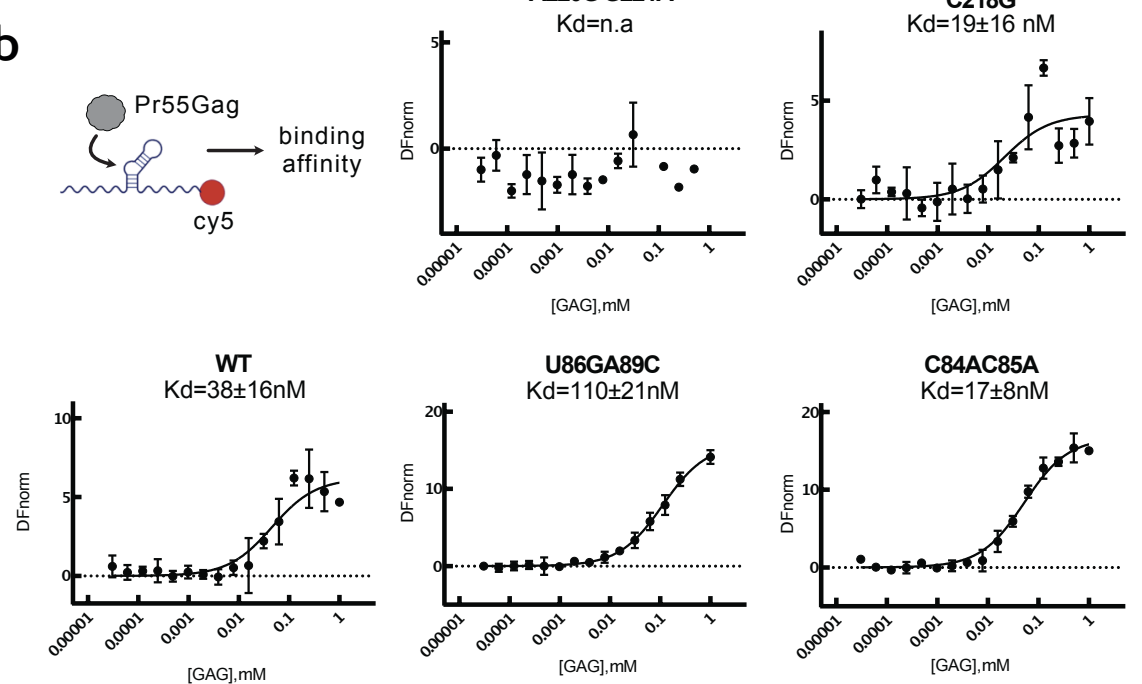

Pairwise competition assay Packaging efficiency

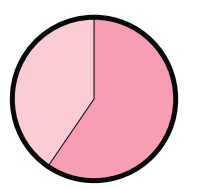

PBS-SL1 interaction

$\square$ C218G

$\square$ A220G G221A

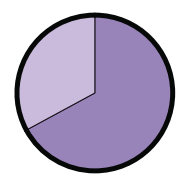

polyA-SL1 interaction

$\square$ C84A C85A

$\square$ U86G A89C

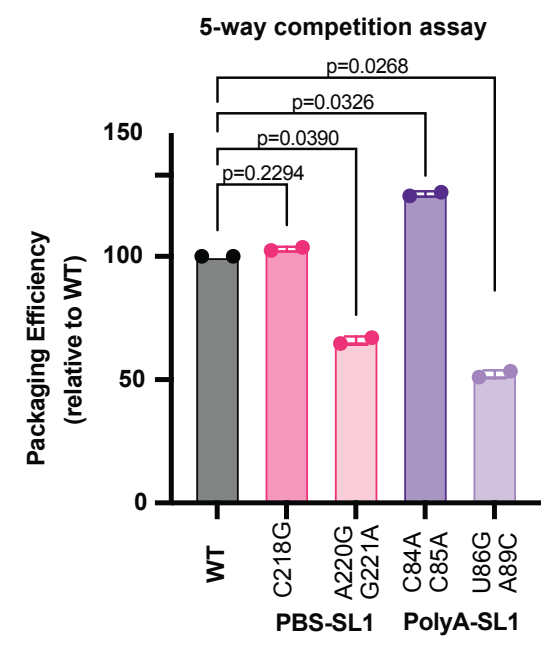

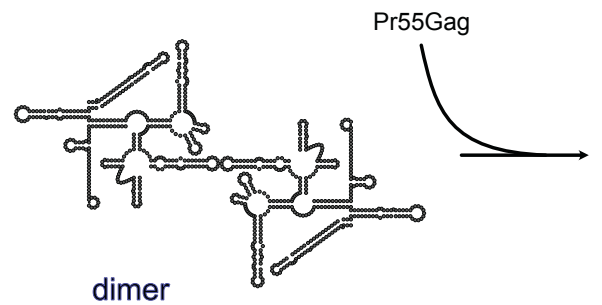

packaging

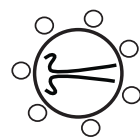




\section{$100 \quad$ Figure 7}

101 PBS regulates dimerization and Pr55 ${ }^{\mathrm{Gag}}$ binding. (a) PBS targeting oligos can trigger dimerization 102 of a 3G RNA. cPBS(182-199) disrupts the TAR-PBS interaction leading to the formation of a higher 103 order RNA structure. cPBS(199-216) disrupts the PAS-antiPAS stem and enhances dimerization. 104 The effects of both oligos are additive (b) Mutations targeting the polyA-SL1 and PBS-SL1 105 interaction affect $\operatorname{Pr} 55^{\text {Gag }}$ binding as measured by microscale thermophoresis (MST) (c) 106 Competition assays to measure the relative effects of mutations on genome packaging into virions.

107 Two-way competition assays show that dimer promoting mutations C218G and C84A-C85A are 108 enhanced in genome packaging compared to monomer promoting mutations A220G-G221A and 109 U86G A89C. Five-way competition assays between wild-type (WT) HIV-1 and mutants show that 110 dimer promoting mutants are packaged similar or better than WT, whereas monomer promoting 111 mutants are packaged less efficiently than WT. Experiments were performed in duplicate. Statistical 112 test was a one-way ANOVA with Dunnett's correction for multiple comparisons (d) Model showing 113 how the binding of host factors can regulate viral replication, in part, through re-modelling RNA 114 structure. 


\section{Supplementary Files}

This is a list of supplementary files associated with this preprint. Click to download.

- SupplementaryFigures.pdf

- SupplementaryNote.pdf

- Conservation.zip

- 1dstructure.zip

- DMStables.zip

- Kdimertables.zip

- DMSplots.zip

- Kdimerplots.zip

- XMLDMSreactivities.zip

- deltaSHAPE.zip

- 2dstructureinference.zip

- MIMEAnToKdResults.zip 